\title{
Assessment of substituent effects and antimicrobial activities of some 2',5'-dimethyl phenyl chalcones
}

\author{
S. John Joseph ${ }^{1}$, D. Kamalakkannan ${ }^{1}$, R. Arulkumaran ${ }^{1}$, S. P. Sakthinathan ${ }^{1}$, \\ R. Suresh ${ }^{1}$, R. Sundararajan ${ }^{1}$, S. Vijayakumar ${ }^{1}$, K. Ranganathan ${ }^{1}$, \\ G. Vanangamudi ${ }^{1, *}, \mathbf{G}$. Thirunarayanan ${ }^{2}$ \\ ${ }^{1} P G$ \& Research Department of Chemistry, Government Arts College, C-Mutlur, \\ Chidambaram - 608102, India \\ ${ }^{2}$ Department of Chemistry, Annamalai University, Annamalainagar - 608002, India \\ *E-mail address: drgvsibi@gmail.com
}

\begin{abstract}
Some 2',5'-dimethyl phenyl chalcones have been synthesized by Crossed-Aldol condensation between 2,5-dimethyl acetophenone and various substituted benzaldehydes using catalytic amount of sodium hydroxide and ethanol. The yields of the chalcones are more than $93 \%$. The purities of these chalcones have been checked by their physical constants, UV, IR, NMR and MASS spectral data. The spectral data of these chalcones have been correlated with Hammett sigma constants, $\mathrm{F}$ and $\mathrm{R}$ parameters using single and multi-linear regression analysis. From the results of statistical analysis, the effects of substituents on the spectral group frequencies have been discussed. The anti-microbial activities of these chalcones have been evaluated using Bauer-Kirby method.
\end{abstract}

Keywords: Substituted styryl 2',5'-dimethyl phenyl chalcones; UV spectra; IR spectra; NMR spectra; Substituent effects; Antimicrobial activities

\section{INTRODUCTION}

Chalcones are, $\alpha, \beta$ unsaturated ketones, possess methylene structural moieties and they belongs to biomolecules. Many alkyl-alkyl, alkyl-aryl and aryl-aryl categories of chalcones have been synthesized [1] and extracted from natural plants [2] by organic chemists. Various methods available for synthesizing chalcones such as Aldol, Crossed-Aldol, Claisen-Schmidt, Knovenagal reactions, Greener methods-Grinding of reactants, solvent free and oxides of nanoparticles with microwave heating. Also microwave assisted solvent free Aldol and Crossed-Aldol condensation [3-5] was useful for synthesis of carbonyl compounds. Due to C$\mathrm{C}$ single bond rotation [6] of carbonyl and alkene carbons, they exist as Es-cis and s-trans and $Z s$-cis and $Z s$-trans conformers. These structural conformers of chalcones have been confirmed by NMR and IR spectroscopy.

Compounds with the backbone of chalcone possesses various biological activities such as anti-microbial [7], anti-inflammatory [8], analgesic [9], anti-ulcerative [10], immunemodulatory [11], anti-malarial [12], anti-cancer [13], anti-viral [14], anti-leishmanial [15], anti-oxidant [16], anti-tubercular [17], anti-hyperglycemic [18] etc. A compound having anti- 
oxidant activity prevents and counteracts the damage of the human tissue by the normal effects of physiological oxidation [19].

Presence of the reactive keto group and the vinylenic group in the chalcone and their analogues possesses the antioxidant activity [20]. Compounds that associated with the antioxidant properties are hydroxyl and phenyl group. Oxidative stress which is caused by the free radical damage is help to deal with the antioxidants [21]. Spectroscopic data is useful for study of effects of substituents on the specified spectral group frequencies using Hammett substituent constants, $\mathrm{F}$ and $\mathrm{R}$ parameters by single and multi-regression analysis. From the results the ground state molecular equilibration can be predicted. Recently Subramanian et. al. $[22,23]$ has studied the synthesis and spectral correlations of some heterocyclic chalcones and they observed satisfactory correlations.

In their studies, they observed satisfactory and good correlations for each spectral data with Hammett substituent constants, $\mathrm{F}$ and $\mathrm{R}$ parameters. Chalcones possess various multipronged activities such as antimicrobial [7], antidepressants [26], antiplosmodial [27], anti-aids [28] and insect antifeedant activities [24,25]. In the present investigation, the authors have synthesized a series of some substituted styryl 2,5-dimethyl pheny chalcones, studied the spectral correlations and to evaluate the antimicrobial activities using Bauer-Kirby [29] method.

\section{EXPERIMENTAL}

\section{1. General}

All chemicals used were purchased from Sigma-Aldrich and E-Merck chemical companies. Melting points of all chalcones were determined in open glass capillaries on Mettler FP51 melting point apparatus and are uncorrected. The ultraviolet spectrophotometer was utilized for recording the absorption maxima $(\lambda \max , \mathrm{nm})$, of all chalcones in spectral grade solvent. Infrared spectra $\left(\mathrm{KBr}, 4000-400 \mathrm{~cm}^{-1}\right)$ were recorded AVATAR-300 Fourier transform spectrophotometer.

BRUKER AVANCE III-500 MHz NMR spectrometer was used for recording NMR spectra operating at $500 \mathrm{MHz}$ for ${ }^{1} \mathrm{H}$ spectra and $125.46 \mathrm{MHz}$ for ${ }^{13} \mathrm{C}$ spectra in $\mathrm{CDCl}_{3}$ solvent using TMS as internal standard. Mass spectra of all chalcones were recorded on SHIMADZU spectrometer using chemical ionization technique.

\section{2. Synthesis of Chalcones}

A solution of benzaldehyde $(0.01 \mathrm{~mol})$ and 2,5 -dimethyl acetophenone $(0.01 \mathrm{~mol})$, sodium hydroxide $(0.5 \mathrm{~g})$ and $10 \mathrm{ml}$ of ethanol was shaken occasionally for 1 hour [30]. After the completion of the reaction, as monitored by TLC, the mixture was cooled at room temperature. The resulting precipitate was filtered and washed with cold water. The product appeared as light green solid. Then this was recrystallised using ethanol to obtain light green glittering solid melting at $116-117^{\circ} \mathrm{C}$. The physical constants, analytical and mass fragments were presented in Table 1. 


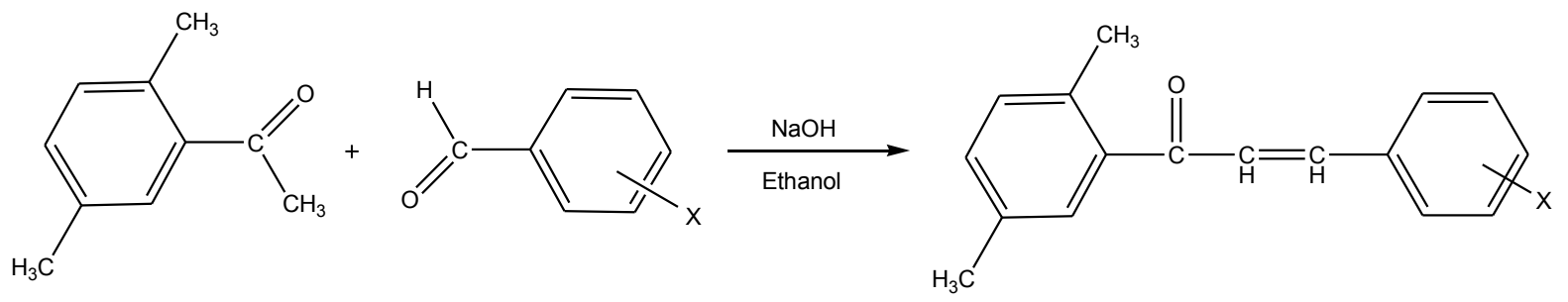

Where $\mathrm{X}=\mathrm{H}, 4-\mathrm{Br}, 2-\mathrm{Cl}, 4-\mathrm{Cl}, 4-\mathrm{F}, 4-\mathrm{OCH}_{3}, 4-\mathrm{CH}_{3}, 3-\mathrm{NO}_{2}, 4-\mathrm{NO}_{2}$

Scheme 1. Synthesis of substituted styryl 2',5'-dimethyl phenyl ketones.

Table 1. Physical constants and mass spectral data of substituted styryl 2',5'-dimethyl phenyl ketones.

\begin{tabular}{|c|c|c|c|c|c|c|}
\hline Entry & $\mathbf{X}$ & $\mathbf{M} . \mathbf{F}$. & $\mathbf{M} . \mathbf{W}$. & $\begin{array}{c}\text { Yield } \\
\mathbf{( \% )}\end{array}$ & $\mathbf{m . p .}\left({ }^{\circ} \mathbf{C}\right)$ & Mass (m/z) \\
\hline 1 & $\mathrm{H}$ & $\mathrm{C}_{17} \mathrm{H}_{16} \mathrm{O}$ & 236 & 93 & $116-117$ & $236[\mathrm{M}+]$ \\
\hline 2 & $4-\mathrm{Br}$ & $\mathrm{C}_{17} \mathrm{H}_{15} \mathrm{BrO}$ & 315 & 91 & $131-133$ & $315[\mathrm{M}+], 317[\mathrm{M} 2+]$ \\
\hline 3 & $2-\mathrm{Cl}$ & $\mathrm{C}_{17} \mathrm{H}_{15} \mathrm{ClO}$ & 270 & 88 & $108-109$ & $270[\mathrm{M}+], 272[\mathrm{M} 2+]$ \\
\hline 4 & $4-\mathrm{Cl}$ & $\mathrm{C}_{17} \mathrm{H}_{15} \mathrm{ClO}$ & 270 & 89 & $101-103$ & $270[\mathrm{M}+], 272[\mathrm{M} 2+]$ \\
\hline 5 & $4-\mathrm{F}$ & $\mathrm{C}_{17} \mathrm{H}_{15} \mathrm{FO}$ & 254 & 88 & $94-96$ & $254[\mathrm{M}+], 256[\mathrm{M} 2+]$ \\
\hline 6 & $4-\mathrm{OCH}_{3}$ & $\mathrm{C}_{18} \mathrm{H}_{18} \mathrm{O}_{2}$ & 267 & 87 & $96-98$ & $267[\mathrm{M}+]$ \\
\hline 7 & $4-\mathrm{CH}_{3}$ & $\mathrm{C}_{18} \mathrm{H}_{18} \mathrm{O}$ & 250 & 90 & $111-113$ & $250[\mathrm{M}+]$ \\
\hline 8 & $3-\mathrm{NO}_{2}$ & $\mathrm{C}_{17} \mathrm{H}_{15} \mathrm{NO}_{3}$ & 281 & 89 & $145-147$ & $281[\mathrm{M}+]$ \\
\hline 9 & $4-\mathrm{NO}_{2}$ & $\mathrm{C}_{17} \mathrm{H}_{15} \mathrm{NO}_{3}$ & 281 & 84 & $139-141$ & $281[\mathrm{M}+]$ \\
\hline
\end{tabular}

\section{RESULTS AND DISCUSSION}

In the present study the authors have studied the effects of substituents on the spectral data such as ultraviolet absorption maxima $(\lambda \max , \mathrm{nm})$, infrared spectral carbonyl and deformation modes $\left(v, \mathrm{~cm}^{-1}\right)$, the chemical shifts $(\delta, \mathrm{ppm})$ of $\alpha, \beta$ protons, and carbonyl carbons of synthesized 2,5-dimethyl phenyl chalcones with Hammett substituent constants, F and $\mathrm{R}$ parameters using single and multi-linear regression analysis for predicting the reactivity on the group frequencies.

\section{1. UV spectral study}

The absorption maxima ( $\lambda \max , \mathrm{nm})$ of synthesized 2,5-dimethyl phenyl chalcones were assigned and presented in Table 2 . These absorption maxima $(\lambda \max , \mathrm{nm})$ of these chalcones 
were correlated with Hammett substituent constants and F and R parameters using single and multi-linear regression analysis [1,4,22-23,31-38]. Hammett correlation involving the group frequencies and absorption maxima, the form of the Hammett equation employed is

$$
\lambda \max =\rho \sigma+\lambda_{0}
$$

The results of statistical analysis are presented in Table 3. From the Table 3, the results of statistical analysis [1,4,22-23,31-38] of these UV absorption maxima ( $\lambda$ max, nm) data with Hammett substituent constants, except that with $4-\mathrm{NO}_{2}$ substituent have shown satisfactory correlations with Hammett substituent constant $\sigma(\mathrm{r}=0.905)$ and except those with 4-Br and 4- $\mathrm{NO}_{2}$ substituents have shown satisfactory correlations with Hammett substituent constant $\sigma^{+}(\mathrm{r}=0.906)$.

The remaining Hammett substituent constants $\sigma_{\mathrm{I}}, \sigma_{\mathrm{R}}$ and $F$ and $R$ parameters have shown poor correlations $(\mathrm{r}<0.900)$. This is due to the weak inductive, field and resonance effects of the substituents for predicting the reactivity on the absorption and is associated with the resonance conjugated structure as shown in Fig. 1.

In single parameter correlation, the Hammett substituents constants not obeyed in the regression. While seeking these parameters in multi-regression, with $\mathrm{F}$ and $\mathrm{R}$ SwainLupton's [39] constants, they gave satisfactory correlations. The multi correlation equations are given in (2) and (3).

$$
\begin{gathered}
\lambda \max (\mathrm{nm})=307.76( \pm 9.953)-15.847( \pm 19.964) \sigma_{\mathrm{I}}-28.122( \pm 23.011) \sigma_{\mathrm{R}} \\
(R=0.953, \mathrm{n}=9, \mathrm{P}>95 \%) \\
\lambda \max (\mathrm{nm})=306.62( \pm 9.165)-16.506( \pm 18.055) \mathrm{F}-29.950( \pm 18.373) \mathrm{R} \\
(R=0.960, \mathrm{n}=9, \mathrm{P}>95 \%)
\end{gathered}
$$

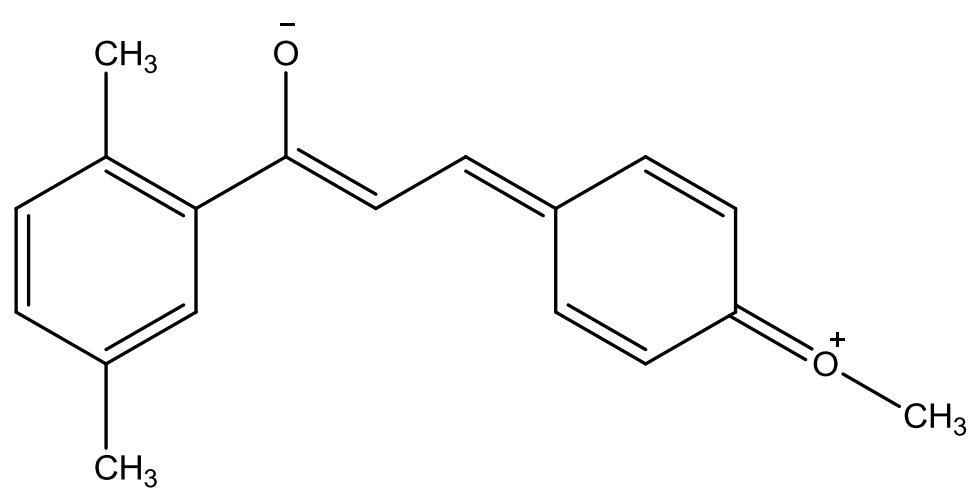

Fig. 1. The resonance- conjugated structure. 
Table 2. The ultraviolet absorption maxima $(\lambda \max , \mathrm{nm})$, infrared absorptions $\left(v, \mathrm{~cm}^{-1}\right)$ and NMR chemical shifts $(\delta, \mathrm{ppm})$ of substituted styryl 2',5'-dimethyl phenyl chalcones.

\begin{tabular}{|c|c|c|c|c|c|c|c|c|c|c|c|c|c|}
\hline$\underset{=}{\stackrel{Z}{Z}}$ & $x$ & 站 & نُ & $e^{\underline{\Sigma}}$ & $\stackrel{乛}{ت}$ & $\underset{0}{5}$ & $\underbrace{I I}_{0}$ & $\bigcup_{0}^{\delta}$ & $\frac{\pi}{0}$ & $\frac{\infty}{10}$ & $\bigcup_{0}^{\infty}$ & $\bigcup_{0}^{\infty}$ & Oِ \\
\hline 1 & $I$ & $\begin{array}{l}\infty \\
\infty \\
\dot{8} \\
\end{array}$ & $\begin{array}{l}\infty \\
\infty \\
\infty \\
0 \\
0\end{array}$ & $\begin{array}{l}\hat{n} \\
\tilde{n}\end{array}$ & $\begin{array}{l}\text { ก̦ } \\
\stackrel{n}{n} \\
=\end{array}$ & 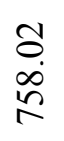 & $\begin{array}{l}\stackrel{P}{2} \\
0 \\
0 \\
0\end{array}$ & $\begin{array}{l}\bar{\sim} \\
\text { ஸூ } \\
n\end{array}$ & $\begin{array}{l}\infty \\
0 \\
\end{array}$ & $\begin{array}{l}8 \\
\\
\end{array}$ & $\begin{array}{l}\infty \\
\dot{J} \\
\stackrel{+}{ }\end{array}$ & $\begin{array}{l}0 \\
\stackrel{0}{0} \\
\stackrel{D}{ \pm}\end{array}$ & $\begin{array}{l}\stackrel{a}{0} \\
\dot{\sigma}\end{array}$ \\
\hline 2 & $\dot{\bar{\varphi}}$ & $\begin{array}{l}\stackrel{+}{+} \\
\dot{0} \\
\stackrel{0}{0}\end{array}$ & $\begin{array}{l}\tilde{y} \\
0 \\
0 \\
0\end{array}$ & 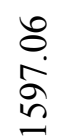 & $\begin{array}{l}2 \\
\hat{\sigma} \\
\emptyset \\
=\end{array}$ & $\begin{array}{l}\text { ஜீ } \\
\text { જి }\end{array}$ & $\begin{array}{l}\vec{\nabla} \\
\infty \\
0 \\
0\end{array}$ & $\begin{array}{l}0 \\
\circ \\
\dot{0} \\
\infty\end{array}$ & $\stackrel{n}{\check{n}}$ & 定 & $\begin{array}{l}\stackrel{ \pm}{\infty} \\
\stackrel{ \pm}{J}\end{array}$ & $\begin{array}{l}\infty \\
\stackrel{\infty}{\Xi} \\
\stackrel{\Xi}{\Xi}\end{array}$ & $\stackrel{n}{\stackrel{2}{\infty}}$ \\
\hline 3 & $\begin{array}{l}\vec{U} \\
\stackrel{\sim}{N}\end{array}$ & $\begin{array}{l}\stackrel{+}{+} \\
\dot{\gamma} \\
\stackrel{2}{2}\end{array}$ & $\begin{array}{l}\infty \\
\infty \\
\infty \\
0 \\
0\end{array}$ & $\begin{array}{l}n \\
\stackrel{n}{2} \\
\stackrel{n}{n}\end{array}$ & $\begin{array}{l}\hat{\sigma} \\
\dot{0} \\
=\end{array}$ & $\begin{array}{l}\checkmark \\
0 \\
\infty \\
\curvearrowright \\
\sim\end{array}$ & 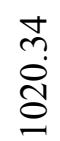 & $\begin{array}{l}0 \\
\infty \\
\stackrel{i}{n} \\
\text { n. }\end{array}$ & 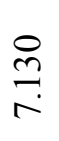 & $\underset{\sim}{\stackrel{f}{r}}$ & $\begin{array}{l}n \\
\stackrel{0}{0} \\
\stackrel{1}{n}\end{array}$ & $\begin{array}{l}\infty \\
\stackrel{\sim}{\Xi} \\
\underset{\Xi}{\Xi}\end{array}$ & 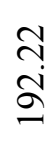 \\
\hline 4 & $\begin{array}{l}\vec{I} \\
\dot{t}\end{array}$ & $\begin{array}{l}8 \\
\dot{0} \\
\infty \\
0 \\
0\end{array}$ & $\begin{array}{l}0 \\
n \\
0 \\
0 \\
0\end{array}$ & 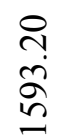 & $\begin{array}{l}0 \\
\hat{\sigma} \\
0 \\
=\end{array}$ & $\frac{\infty}{\stackrel{\infty}{\infty}}$ & $\begin{array}{l}\stackrel{P}{2} \\
0 \\
0\end{array}$ & $\frac{\partial}{\frac{\alpha}{+}}$ & $\frac{\stackrel{N}{\sim}}{\sim}$ & $\begin{array}{l}n \\
\stackrel{n}{r} \\
r\end{array}$ & $\begin{array}{l}8 \\
0 \\
\text { 이 }\end{array}$ & $\begin{array}{l}\infty \\
\stackrel{\infty}{\Xi} \\
\stackrel{\sim}{ \pm}\end{array}$ & $\stackrel{\vartheta}{\stackrel{\vartheta}{a}}$ \\
\hline 5 & $\frac{1}{\dot{\gamma}}$ & $\begin{array}{l}\stackrel{8}{0} \\
\dot{0} \\
\text { D }\end{array}$ & $\begin{array}{l}\mathbb{b} \\
i \\
0 \\
0\end{array}$ & $\begin{array}{l}\text { กิ } \\
\text { ñ } \\
\hat{n}\end{array}$ & $\begin{array}{l}\infty \\
\stackrel{\infty}{0} \\
\stackrel{0}{=}\end{array}$ & $\begin{array}{l}\infty \\
\stackrel{\infty}{\infty}\end{array}$ & $\begin{array}{l}\vec{J} \\
\bar{\sigma} \\
\sigma\end{array}$ & $\begin{array}{l}\stackrel{y}{~} \\
\text { } \\
\stackrel{8}{n}\end{array}$ & $\stackrel{ }{I}$ & $\begin{array}{l}\infty \\
\stackrel{\infty}{+} \\
\stackrel{r}{r}\end{array}$ & $\begin{array}{l}\infty \\
\stackrel{\sim}{\sim} \\
\stackrel{\sim}{\sim}\end{array}$ & $\begin{array}{l}\vec{\sim} \\
\stackrel{\Xi}{ \pm}\end{array}$ & $\begin{array}{l}\stackrel{\sim}{2} \\
\stackrel{2}{2}\end{array}$ \\
\hline 6 & 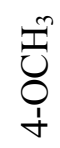 & $\begin{array}{c}\text { ָे } \\
\text { } \\
\text { m}\end{array}$ & $\begin{array}{l}\infty \\
\infty \\
\infty \\
0 \\
0\end{array}$ & $\begin{array}{l}\stackrel{a}{+} \\
\dot{\infty} \\
\infty \\
n\end{array}$ & $\begin{array}{l}n \\
\stackrel{0}{\sigma} \\
=\end{array}$ & $\begin{array}{l}\stackrel{0}{0} \\
\stackrel{0}{\infty}\end{array}$ & $\begin{array}{l}0 \\
\stackrel{+}{+} \\
o\end{array}$ & $\frac{\infty}{\stackrel{\infty}{\sigma}}$ & $\begin{array}{l}\stackrel{\infty}{\mathbb{O}} \\
\stackrel{\sim}{\sim}\end{array}$ & $\underset{r}{\stackrel{g}{f}}$ & 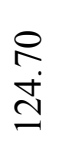 & 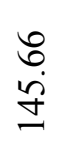 & $\begin{array}{l}\dot{\sigma} \\
\text { a }\end{array}$ \\
\hline 7 & 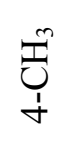 & $\begin{array}{l}8 \\
\stackrel{8}{m} \\
\frac{1}{m}\end{array}$ & $\begin{array}{l}\mathbb{b} \\
i \\
0 \\
0\end{array}$ & $\begin{array}{l}\stackrel{m}{n} \\
\stackrel{n}{n}\end{array}$ & 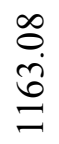 & $\begin{array}{l}\tilde{a} \\
\tilde{n} \\
\kappa\end{array}$ & $\begin{array}{l}\vec{\gamma} \\
\infty \\
0\end{array}$ & $\begin{array}{l}\bar{\sim} \\
\text { ஸे } \\
n\end{array}$ & $\frac{\stackrel{N}{\sim}}{\sim}$ & $\frac{\stackrel{\sim}{\sim}}{\sim}$ & $\begin{array}{l}20 \\
\stackrel{1}{1} \\
\text { In }\end{array}$ & $\begin{array}{l}\infty \\
\infty \\
\stackrel{D}{ \pm}\end{array}$ & $\hat{\grave{g}}$ \\
\hline 8 & $\begin{array}{l}\sigma_{n}^{N} \\
Z_{1}^{\prime}\end{array}$ & $\begin{array}{l}\stackrel{+}{+} \\
\infty \\
\stackrel{\infty}{N}\end{array}$ & $\begin{array}{l}\text { ț } \\
\text { ర్ } \\
0\end{array}$ & 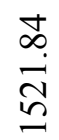 & 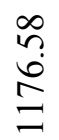 & $\begin{array}{l}\stackrel{\infty}{\infty} \\
\stackrel{\infty}{\infty}\end{array}$ & 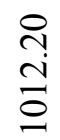 & $\begin{array}{l}\hat{0} \\
\hat{\sigma} \\
n\end{array}$ & ৯े & $\begin{array}{l}\infty \\
n \\
n \\
n\end{array}$ & $\begin{array}{l}\text { ర } \\
\text { ป } \\
\text { I }\end{array}$ & $\begin{array}{l}\text { ō } \\
\stackrel{\text { J }}{ \pm}\end{array}$ & $\begin{array}{l}\stackrel{\sim}{\sim} \\
\stackrel{2}{\Omega}\end{array}$ \\
\hline 9 & 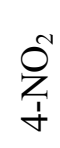 & $\begin{array}{l}\stackrel{+}{+} \\
\dot{m} \\
\dot{m}\end{array}$ & $\begin{array}{l}\overrightarrow{8} \\
8 \\
0\end{array}$ & $\begin{array}{l}0 \\
n \\
\infty \\
\infty \\
n\end{array}$ & $\begin{array}{l}n \\
\stackrel{n}{6} \\
=\end{array}$ & $\begin{array}{l}n \\
\stackrel{1}{\infty} \\
\stackrel{\infty}{0}\end{array}$ & $\begin{array}{l}3 \\
i \\
i \\
0\end{array}$ & 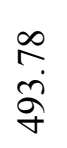 & ণৃ & 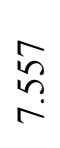 & 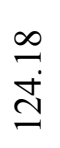 & $\begin{array}{l}\stackrel{\infty}{\perp} \\
\underset{\Xi}{ \pm}\end{array}$ & $=$ \\
\hline
\end{tabular}


Table 3. Results of statistical analysis of ultraviolet absorption maxima $(\lambda \max , \mathrm{nm})$, infrared absorptions $\left(v, \mathrm{~cm}^{-1}\right)$ and NMR chemical shifts $(\delta, \mathrm{ppm})$ of substituted styryl $2^{\prime}, 5^{\prime}$-dimethyl phenyl chalcones with Hammett $\sigma, \sigma^{+}, \sigma_{\mathrm{I}}, \sigma_{\mathrm{R}}$ and $\mathrm{F}$ and R parameters

\begin{tabular}{|c|c|c|c|c|c|c|c|}
\hline Frequency & $\begin{array}{c}\text { Constant } \\
\text { s }\end{array}$ & $\mathbf{r}$ & $\mathbf{I}$ & $\rho$ & $\mathbf{S}$ & $\mathbf{n}$ & Correlated derivatives \\
\hline \multirow{6}{*}{ है } & $\sigma$ & 0.905 & 310.12 & -22.43 & 13.11 & 8 & $\begin{array}{c}\mathrm{H}, 4-\mathrm{Br}, 2-\mathrm{Cl}, 4-\mathrm{Cl}, \\
4-\mathrm{F}, 4-\mathrm{OCH}_{3}, 4-\mathrm{CH}_{3}, \\
3-\mathrm{NO}_{2}\end{array}$ \\
\hline & $\sigma^{+}$ & 0.906 & 308.60 & -19.78 & 11.54 & 7 & $\begin{array}{c}\mathrm{H}, 2-\mathrm{Cl}, 4-\mathrm{Cl}, 4-\mathrm{F}, \\
4-\mathrm{OCH}_{3}, 4-\mathrm{CH}_{3}, 3-\mathrm{NO}_{2}\end{array}$ \\
\hline & $\sigma_{\mathrm{I}}$ & 0.803 & 312.89 & -18.69 & 14.88 & 9 & $\begin{array}{c}\mathrm{H}, 4-\mathrm{Br}, 2-\mathrm{Cl}, 4-\mathrm{Cl}, \\
\text { 4-F, 4-OCH}, 4-\mathrm{CH}_{3}, \\
\text { 3- } \mathrm{NO}_{2}, 4-\mathrm{NO}_{2}\end{array}$ \\
\hline & $\sigma_{\mathrm{R}}$ & 0.834 & 301.35 & -30.25 & 14.00 & 9 & $\begin{array}{c}\mathrm{H}, 4-\mathrm{Br}, 2-\mathrm{Cl}, 4-\mathrm{Cl}, \\
\text { 4-F, 4- } \mathrm{OCH}_{3}, 4-\mathrm{CH}_{3}, \\
\text { 3- } \mathrm{NO}_{2}, 4-\mathrm{NO}_{2}\end{array}$ \\
\hline & $\mathrm{F}$ & 0.822 & 311.90 & -15.40 & 15.12 & 9 & $\begin{array}{c}\mathrm{H}, 4-\mathrm{Br}, 2-\mathrm{Cl}, 4-\mathrm{Cl}, \\
\text { 4-F, 4- } \mathrm{OCH}_{3}, 4-\mathrm{CH}_{3}, \\
\text { 3- } \mathrm{NO}_{2}, 4-\mathrm{NO}_{2}\end{array}$ \\
\hline & $\mathrm{R}$ & 0.845 & 300.08 & -29.32 & 13.43 & 9 & $\begin{array}{c}\mathrm{H}, 4-\mathrm{Br}, 2-\mathrm{Cl}, 4-\mathrm{Cl}, \\
4-\mathrm{F}, 4-\mathrm{OCH}_{3}, 4-\mathrm{CH}_{3}, \\
\text { 3- } \mathrm{NO}_{2}, 4-\mathrm{NO}_{2}\end{array}$ \\
\hline \multirow{3}{*}{$\underbrace{\substack{u \\
\vdots}}$} & $\sigma$ & 0.817 & 1661.88 & 1.634 & 3.63 & 9 & $\begin{array}{c}\mathrm{H}, 4-\mathrm{Br}, 2-\mathrm{Cl}, 4-\mathrm{Cl}, \\
\text { 4-F, } 4-\mathrm{OCH}_{3}, 4-\mathrm{CH}_{3}, \\
\text { 3- } \mathrm{NO}_{2}, 4-\mathrm{NO}_{2}\end{array}$ \\
\hline & $\sigma^{+}$ & 0.822 & 1661.98 & 1.505 & 3.59 & 9 & $\begin{array}{c}\mathrm{H}, 4-\mathrm{Br}, 2-\mathrm{Cl}, 4-\mathrm{Cl}, \\
4-\mathrm{F}, 4-\mathrm{OCH}_{3}, 4-\mathrm{CH}_{3}, \\
\text { 3- } \mathrm{NO}_{2}, 4-\mathrm{NO}_{2}\end{array}$ \\
\hline & $\sigma_{\mathrm{I}}$ & 0.826 & 1660.83 & 3.558 & 3.55 & 9 & $\begin{array}{c}\mathrm{H}, 4-\mathrm{Br}, 2-\mathrm{Cl}, 4-\mathrm{Cl}, 4-\mathrm{F}, \\
4-\mathrm{OCH}_{3}, 4-\mathrm{CH}_{3},\end{array}$ \\
\hline
\end{tabular}




\begin{tabular}{|c|c|c|c|c|c|c|c|}
\hline & & & & & & & $3-\mathrm{NO}_{2}, 4-\mathrm{NO}_{2}$ \\
\hline & $\sigma_{\mathrm{R}}$ & 0.802 & 1662.27 & 0.454 & 3.68 & 9 & $\begin{array}{c}\mathrm{H}, 4-\mathrm{Br}, 2-\mathrm{Cl}, 4-\mathrm{Cl}, 4-\mathrm{F}, \\
4-\mathrm{OCH}_{3}, 4-\mathrm{CH}_{3}, \\
3-\mathrm{NO}_{2}, 4-\mathrm{NO}_{2}\end{array}$ \\
\hline & $\mathrm{F}$ & 0.822 & 1660.94 & 3.148 & 3.57 & 9 & $\begin{array}{c}\mathrm{H}, 4-\mathrm{Br}, 2-\mathrm{Cl}, 4-\mathrm{Cl}, 4-\mathrm{F}, \\
4-\mathrm{OCH}_{3}, 4-\mathrm{CH}_{3}, \\
3-\mathrm{NO}_{2}, 4-\mathrm{NO}_{2}\end{array}$ \\
\hline & $\mathrm{R}$ & 0.707 & 1662.23 & 0.097 & 3.68 & 9 & $\begin{array}{c}\mathrm{H}, 4-\mathrm{Br}, 2-\mathrm{Cl}, 4-\mathrm{Cl}, 4-\mathrm{F}, \\
4-\mathrm{OCH}_{3}, 4-\mathrm{CH}_{3}, \\
3-\mathrm{NO}_{2}, 4-\mathrm{NO}_{2}\end{array}$ \\
\hline \multirow{6}{*}{ 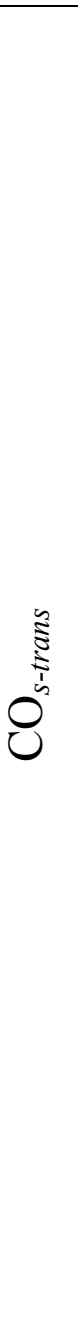 } & $\sigma$ & 0.905 & 1591.50 & -38.03 & 20.62 & 7 & $\begin{array}{l}\mathrm{H}, 4-\mathrm{Br}, 2-\mathrm{Cl}, 4-\mathrm{Cl}, \\
4-\mathrm{F}, 4-\mathrm{OCH}_{3}, 4-\mathrm{CH}_{3}\end{array}$ \\
\hline & $\sigma^{+}$ & 0.904 & 1586.83 & -19.34 & 23.11 & 7 & $\begin{array}{l}\mathrm{H}, 4-\mathrm{Br}, 2-\mathrm{Cl}, 4-\mathrm{Cl}, \\
4-\mathrm{F}, 4-\mathrm{OCH}_{3}, 4-\mathrm{CH}_{3}\end{array}$ \\
\hline & $\sigma_{\mathrm{I}}$ & 0.801 & 1598.71 & -38.20 & 23.10 & 9 & $\begin{array}{c}\mathrm{H}, 4-\mathrm{Br}, 2-\mathrm{Cl}, 4-\mathrm{Cl}, 4-\mathrm{F}, \\
\text { 4- } \mathrm{OCH}_{3}, 4-\mathrm{CH}_{3}, \\
\text { 3- } \mathrm{NO}_{2}, 4-\mathrm{NO}_{2}\end{array}$ \\
\hline & $\sigma_{\mathrm{R}}$ & 0.855 & 1576.00 & -55.67 & 21.63 & 9 & $\begin{array}{c}\mathrm{H}, 4-\mathrm{Br}, 2-\mathrm{Cl}, 4-\mathrm{Cl}, 4-\mathrm{F}, \\
4-\mathrm{OCH}_{3}, 4-\mathrm{CH}_{3}, \\
3-\mathrm{NO}_{2}, 4-\mathrm{NO}_{2}\end{array}$ \\
\hline & $\mathrm{F}$ & 0.834 & 1597.38 & -33.22 & 23.54 & 9 & $\begin{array}{c}\mathrm{H}, 4-\mathrm{Br}, 2-\mathrm{Cl}, 4-\mathrm{Cl}, 4-\mathrm{F}, \\
4-\mathrm{OCH}_{3}, 4-\mathrm{CH}_{3}, \\
3-\mathrm{NO}_{2}, 4-\mathrm{NO}_{2}\end{array}$ \\
\hline & $\mathrm{R}$ & 0.845 & 1575.72 & -43.20 & 22.30 & 9 & $\begin{array}{c}\mathrm{H}, 4-\mathrm{Br}, 2-\mathrm{Cl}, 4-\mathrm{Cl}, \\
\text { 4-F, 4- } \mathrm{OCH}_{3},\end{array}$ \\
\hline
\end{tabular}




\begin{tabular}{|c|c|c|c|c|c|c|c|}
\hline & & & & & & & $4-\mathrm{CH}_{3}, 3-\mathrm{NO}_{2}, 4-\mathrm{NO}_{2}$ \\
\hline \multirow{6}{*}{$\stackrel{8}{0}$} & $\sigma$ & 0.905 & 1163.51 & 7.516 & 4.72 & 7 & $\begin{array}{l}\text { 4- } \mathrm{Br}, 2-\mathrm{Cl}, 4-\mathrm{Cl}, 4-\mathrm{F}, \\
4-\mathrm{OCH}_{3}, 4-\mathrm{CH}_{3}, 3-\mathrm{NO}_{2}\end{array}$ \\
\hline & $\sigma^{+}$ & 0.905 & 1164.21 & 5.365 & 4.73 & 6 & $\begin{array}{c}\text { 4- } \mathrm{Br}, 2-\mathrm{Cl}, 4-\mathrm{Cl}, 4-\mathrm{F}, \\
\text { 4- } \mathrm{OCH}_{3}, 4-\mathrm{CH}_{3}\end{array}$ \\
\hline & $\sigma_{\mathrm{I}}$ & 0.905 & 1160.82 & 10.83 & 4.68 & 7 & $\begin{array}{c}\mathrm{H}, 4-\mathrm{Br}, 2-\mathrm{Cl}, 4-\mathrm{Cl}, 4-\mathrm{F}, \\
4-\mathrm{OCH}_{3}, 4-\mathrm{CH}_{3},\end{array}$ \\
\hline & $\sigma_{\mathrm{R}}$ & 0.830 & 1166.01 & 7.056 & 5.29 & 9 & $\begin{array}{c}\mathrm{H}, 4-\mathrm{Br}, 2-\mathrm{Cl}, 4-\mathrm{Cl}, 4-\mathrm{F}, \\
4-\mathrm{OCH}_{3}, 4-\mathrm{CH}_{3}, \\
3-\mathrm{NO}_{2}, 4-\mathrm{NO}_{2}\end{array}$ \\
\hline & $\mathrm{F}$ & 0.844 & 1161.49 & 8.692 & 4.96 & 9 & $\begin{array}{c}\mathrm{H}, 4-\mathrm{Br}, 2-\mathrm{Cl}, 4-\mathrm{Cl}, 4-\mathrm{F}, \\
4-\mathrm{OCH}_{3}, 4-\mathrm{CH}_{3}, \\
3-\mathrm{NO}_{2}, 4-\mathrm{NO}_{2}\end{array}$ \\
\hline & $\mathrm{R}$ & 0.834 & 1166.20 & 6.258 & 5.26 & 9 & $\begin{array}{c}\mathrm{H}, 4-\mathrm{Br}, 2-\mathrm{Cl}, 4-\mathrm{Cl}, 4-\mathrm{F}, \\
4-\mathrm{OCH}_{3}, 4-\mathrm{CH}_{3}, \\
3-\mathrm{NO}_{2}, 4-\mathrm{NO}_{2}\end{array}$ \\
\hline \multirow{3}{*}{$\overbrace{}^{8}$} & $\sigma$ & 0.838 & 785.05 & 30.22 & 28.02 & 9 & $\begin{array}{c}\mathrm{H}, 4-\mathrm{Br}, 2-\mathrm{Cl}, 4-\mathrm{Cl}, 4-\mathrm{F}, \\
4-\mathrm{OCH}_{3}, 4-\mathrm{CH}_{3}, \\
\text { 3- } \mathrm{NO}_{2}, 4-\mathrm{NO}_{2}\end{array}$ \\
\hline & $\sigma^{+}$ & 0.807 & 790.41 & 4.186 & 30.30 & 9 & $\begin{array}{c}\mathrm{H}, 4-\mathrm{Br}, 2-\mathrm{Cl}, 4-\mathrm{Cl}, 4-\mathrm{F}, \\
4-\mathrm{OCH}_{3}, 4-\mathrm{CH}_{3}, \\
3-\mathrm{NO}_{2}, 4-\mathrm{NO}_{2}\end{array}$ \\
\hline & $\sigma_{\mathrm{I}}$ & 0.866 & 763.48 & 71.44 & 23.22 & 9 & $\begin{array}{c}\mathrm{H}, 4-\mathrm{Br}, 2-\mathrm{Cl}, 4-\mathrm{Cl}, 4-\mathrm{F}, \\
4-\mathrm{OCH}_{3}, 4-\mathrm{CH}_{3}, \\
3-\mathrm{NO}_{2}, 4-\mathrm{NO}_{2}\end{array}$ \\
\hline
\end{tabular}




\begin{tabular}{|c|c|c|c|c|c|c|c|}
\hline & $\sigma_{\mathrm{R}}$ & 0.822 & 790.04 & -6.844 & 30.34 & 9 & $\begin{array}{c}\mathrm{H}, 4-\mathrm{Br}, 2-\mathrm{Cl}, 4-\mathrm{Cl}, 4-\mathrm{F}, \\
4-\mathrm{OCH}_{3}, 4-\mathrm{CH}_{3}, \\
3-\mathrm{NO}_{2}, 4-\mathrm{NO}_{2}\end{array}$ \\
\hline & $\mathrm{F}$ & 0.847 & 760.89 & 74.70 & 21.68 & 9 & $\begin{array}{c}\mathrm{H}, 4-\mathrm{Br}, 2-\mathrm{Cl}, 4-\mathrm{Cl}, 4-\mathrm{F}, \\
4-\mathrm{OCH}_{3}, 4-\mathrm{CH}_{3}, \\
3-\mathrm{NO}_{2}, 4-\mathrm{NO}_{2}\end{array}$ \\
\hline & $\mathrm{R}$ & 0.834 & 788.73 & -11.98 & 30.19 & 9 & $\begin{array}{c}\mathrm{H}, 4-\mathrm{Br}, 2-\mathrm{Cl}, 4-\mathrm{Cl}, 4-\mathrm{F}, \\
4-\mathrm{OCH}_{3}, 4-\mathrm{CH}_{3}, \\
3-\mathrm{NO}_{2}, 4-\mathrm{NO}_{2}\end{array}$ \\
\hline \multirow{6}{*}{ 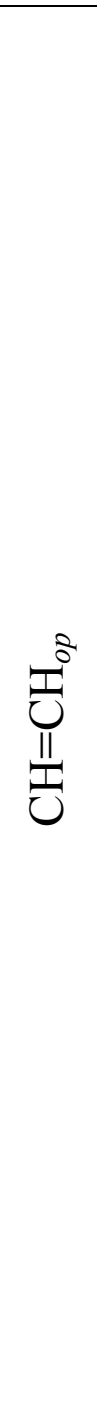 } & $\sigma$ & 0.823 & 1014.85 & -2.427 & 3.882 & 9 & $\begin{array}{c}\mathrm{H}, 4-\mathrm{Br}, 2-\mathrm{Cl}, 4-\mathrm{Cl}, 4-\mathrm{F}, \\
4-\mathrm{OCH}_{3}, 4-\mathrm{CH}_{3}, \\
3-\mathrm{NO}_{2}, 4-\mathrm{NO}_{2}\end{array}$ \\
\hline & $\sigma^{+}$ & 0.815 & 1014.39 & -0.115 & 3.995 & 9 & $\begin{array}{c}\mathrm{H}, 4-\mathrm{Br}, 2-\mathrm{Cl}, 4-\mathrm{Cl}, 4-\mathrm{F}, \\
4-\mathrm{OCH}_{3}, 4-\mathrm{CH}_{3}, \\
3-\mathrm{NO}_{2}, 4-\mathrm{NO}_{2}\end{array}$ \\
\hline & $\sigma_{\mathrm{I}}$ & 0.816 & 1015.28 & -2.364 & 3.942 & 9 & $\begin{array}{c}\mathrm{H}, 4-\mathrm{Br}, 2-\mathrm{Cl}, 4-\mathrm{Cl}, 4-\mathrm{F}, \\
4-\mathrm{OCH}_{3}, 4-\mathrm{CH}_{3}, \\
3-\mathrm{NO}_{2}, 4-\mathrm{NO}_{2}\end{array}$ \\
\hline & $\sigma_{\mathrm{R}}$ & 0.816 & 1013.96 & -2.834 & 3.938 & 9 & $\begin{array}{c}\mathrm{H}, 4-\mathrm{Br}, 2-\mathrm{Cl}, 4-\mathrm{Cl}, 4-\mathrm{F}, \\
4-\mathrm{OCH}_{3}, 4-\mathrm{CH}_{3}, \\
\text { 3- } \mathrm{NO}_{2}, 4-\mathrm{NO}_{2}\end{array}$ \\
\hline & $\mathrm{F}$ & 0.823 & 1015.69 & -3.282 & 3.884 & 9 & $\begin{array}{c}\mathrm{H}, 4-\mathrm{Br}, 2-\mathrm{Cl}, 4-\mathrm{Cl}, 4-\mathrm{F}, \\
4-\mathrm{OCH}_{3}, 4-\mathrm{CH}_{3}, \\
\text { 3- } \mathrm{NO}_{2}, 4-\mathrm{NO}_{2}\end{array}$ \\
\hline & $\mathrm{R}$ & 0.814 & 1013.98 & -2.050 & 3.954 & 9 & $\mathrm{H}, 4-\mathrm{Br}, 2-\mathrm{Cl}, 4-\mathrm{Cl}, 4-\mathrm{F}$ \\
\hline
\end{tabular}




\begin{tabular}{|c|c|c|c|c|c|c|c|}
\hline & & & & & & & $\begin{array}{c}4-\mathrm{OCH}_{3}, 4-\mathrm{CH}_{3}, \\
3-\mathrm{NO}_{2}, 4-\mathrm{NO}_{2}\end{array}$ \\
\hline \multirow{6}{*}{$e_{0}^{8}$} & $\sigma$ & 0.871 & 526.04 & -1.693 & 41.87 & 9 & $\begin{array}{c}\mathrm{H}, 4-\mathrm{Br}, 2-\mathrm{Cl}, 4-\mathrm{Cl}, 4-\mathrm{F}, \\
4-\mathrm{OCH}_{3}, 4-\mathrm{CH}_{3}, \\
\text { 3- } \mathrm{NO}_{2}, 4-\mathrm{NO}_{2}\end{array}$ \\
\hline & $\sigma^{+}$ & 0.813 & 524.21 & 10.15 & 41.51 & 9 & $\begin{array}{c}\mathrm{H}, 4-\mathrm{Br}, 2-\mathrm{Cl}, 4-\mathrm{Cl}, 4-\mathrm{F}, \\
4-\mathrm{OCH}_{3}, 4-\mathrm{CH}_{3}, \\
3-\mathrm{NO}_{2}, 4-\mathrm{NO}_{2}\end{array}$ \\
\hline & $\sigma_{\mathrm{I}}$ & 0.831 & 547.56 & -55.68 & 38.88 & 9 & $\begin{array}{c}\mathrm{H}, 4-\mathrm{Br}, 2-\mathrm{Cl}, 4-\mathrm{Cl}, 4-\mathrm{F}, \\
4-\mathrm{OCH}_{3}, 4-\mathrm{CH}_{3}, \\
3-\mathrm{NO}_{2}, 4-\mathrm{NO}_{2}\end{array}$ \\
\hline & $\sigma_{\mathrm{R}}$ & 0.831 & 533.78 & 56.37 & 39.67 & 9 & $\begin{array}{c}\mathrm{H}, 4-\mathrm{Br}, 2-\mathrm{Cl}, 4-\mathrm{Cl}, 4-\mathrm{F}, \\
4-\mathrm{OCH}_{3}, 4-\mathrm{CH}_{3}, \\
3-\mathrm{NO}_{2}, 4-\mathrm{NO}_{2}\end{array}$ \\
\hline & $\mathrm{F}$ & 0.831 & 549.23 & -58.32 & 38.44 & 9 & $\begin{array}{c}\mathrm{H}, 4-\mathrm{Br}, 2-\mathrm{Cl}, 4-\mathrm{Cl}, 4-\mathrm{F}, \\
4-\mathrm{OCH}_{3}, 4-\mathrm{CH}_{3}, \\
3-\mathrm{NO}_{2}, 4-\mathrm{NO}_{2}\end{array}$ \\
\hline & $\mathrm{R}$ & 0.837 & 536.43 & 56.10 & 38.82 & 9 & $\begin{array}{c}\mathrm{H}, 4-\mathrm{Br}, 2-\mathrm{Cl}, 4-\mathrm{Cl}, 4-\mathrm{F}, \\
4-\mathrm{OCH}_{3}, 4-\mathrm{CH}_{3}, \\
\text { 3- } \mathrm{NO}_{2}, 4-\mathrm{NO}_{2}\end{array}$ \\
\hline \multirow{3}{*}{$\frac{\sigma}{0}$} & $\sigma$ & 0.917 & 7.112 & 0.219 & 0.03 & 7 & $\begin{array}{c}\text { 4-Br, 2-Cl, 4-Cl, 4-F, } \\
\text { 4- } \mathrm{OCH}_{3}, 3-\mathrm{NO}_{2}, 4-\mathrm{NO}_{2}\end{array}$ \\
\hline & $\sigma^{+}$ & 0.907 & 7.134 & 0.143 & 0.05 & 7 & $\begin{array}{l}\mathrm{H}, 4-\mathrm{Cl}, 4-\mathrm{F}, 4-\mathrm{OCH}_{3}, \\
4-\mathrm{CH}_{3}, 3-\mathrm{NO}_{2}, 4-\mathrm{NO}_{2}\end{array}$ \\
\hline & $\sigma_{\mathrm{I}}$ & 0.907 & 7.093 & 0.161 & 0.08 & 7 & 4-Br, 2-Cl, 4-Cl, 4-F, \\
\hline
\end{tabular}




\begin{tabular}{|c|c|c|c|c|c|c|c|}
\hline & & & & & & & $4-\mathrm{OCH}_{3}, 3-\mathrm{NO}_{2}, 4-\mathrm{NO}_{2}$ \\
\hline & $\sigma_{\mathrm{R}}$ & 0.906 & 7.204 & 0.341 & 0.04 & 8 & $\begin{array}{c}\mathrm{H}, 4-\mathrm{Br}, 2-\mathrm{Cl}, 4-\mathrm{Cl} \\
\text { 4- } \mathrm{OCH}_{3}, 4-\mathrm{CH}_{3} \\
3-\mathrm{NO}_{2}, 4-\mathrm{NO}_{2}\end{array}$ \\
\hline & $\mathrm{F}$ & 0.843 & 7.101 & 0.133 & 0.08 & 9 & $\begin{array}{c}\mathrm{H}, 4-\mathrm{Br}, 2-\mathrm{Cl}, 4-\mathrm{Cl}, 4-\mathrm{F}, \\
4-\mathrm{OCH}_{3}, 4-\mathrm{CH}_{3}, \\
3-\mathrm{NO}_{2}, 4-\mathrm{NO}_{2}\end{array}$ \\
\hline & $\mathrm{R}$ & 0.920 & 7.210 & 0.287 & 0.04 & 8 & $\begin{array}{c}\mathrm{H}, 4-\mathrm{Br}, 2-\mathrm{Cl}, 4-\mathrm{Cl}, \\
4-\mathrm{OCH}_{3}, 4-\mathrm{CH}_{3} \\
\text { 3- } \mathrm{NO}_{2}, 4-\mathrm{NO}_{2}\end{array}$ \\
\hline \multirow{6}{*}{$\frac{a}{\frac{\pi}{0}}$} & $\sigma$ & 0.863 & 7.403 & 0.183 & 0.09 & 9 & $\begin{array}{c}\mathrm{H}, 4-\mathrm{Br}, 2-\mathrm{Cl}, 4-\mathrm{Cl}, 4-\mathrm{F}, \\
4-\mathrm{OCH}_{3}, 4-\mathrm{CH}_{3}, \\
3-\mathrm{NO}_{2}, 4-\mathrm{NO}_{2}\end{array}$ \\
\hline & $\sigma^{+}$ & 0.842 & 7.426 & 0.091 & 0.10 & 9 & $\begin{array}{c}\mathrm{H}, 4-\mathrm{Br}, 2-\mathrm{Cl}, 4-\mathrm{Cl}, 4-\mathrm{F}, \\
4-\mathrm{OCH}_{3}, 4-\mathrm{CH}_{3}, \\
3-\mathrm{NO}_{2}, 4-\mathrm{NO}_{2}\end{array}$ \\
\hline & $\sigma_{\mathrm{I}}$ & 0.905 & 7.347 & 0.240 & 0.09 & 7 & $\begin{array}{c}\text { 4- } \mathrm{Br}, 2-\mathrm{Cl}, 4-\mathrm{Cl}, 4-\mathrm{F}, \\
4-\mathrm{OCH}_{3}, 3-\mathrm{NO}_{2}, 4-\mathrm{NO}_{2}\end{array}$ \\
\hline & $\sigma_{\mathrm{R}}$ & 0.835 & 7.465 & 0.173 & 0.10 & 9 & $\begin{array}{c}\mathrm{H}, 4-\mathrm{Br}, 2-\mathrm{Cl}, 4-\mathrm{Cl}, 4-\mathrm{F}, \\
4-\mathrm{OCH}_{3}, 4-\mathrm{CH}_{3}, \\
3-\mathrm{NO}_{2}, 4-\mathrm{NO}_{2}\end{array}$ \\
\hline & $\mathrm{F}$ & 0.905 & 7.350 & 0.221 & 0.09 & 7 & $\begin{array}{c}\text { 4-Br, 2-Cl, 4-Cl, 4- } \\
\mathrm{OCH}_{3} \\
4-\mathrm{CH}_{3}, 3-\mathrm{NO}_{2}, 4-\mathrm{NO}_{2}\end{array}$ \\
\hline & $\mathrm{R}$ & 0.834 & 7.466 & 0.138 & 0.11 & 9 & $\begin{array}{c}\mathrm{H}, 4-\mathrm{Br}, 2-\mathrm{Cl}, 4-\mathrm{Cl}, 4-\mathrm{F}, \\
4-\mathrm{OCH}_{3}, 4-\mathrm{CH}_{3},\end{array}$ \\
\hline
\end{tabular}




\begin{tabular}{|c|c|c|c|c|c|c|c|}
\hline & & & & & & & $3-\mathrm{NO}_{2}, 4-\mathrm{NO}_{2}$ \\
\hline & $\sigma$ & 0.838 & 191.59 & 1.422 & 1.328 & 9 & $\begin{array}{c}\mathrm{H}, 4-\mathrm{Br}, 2-\mathrm{Cl}, 4-\mathrm{Cl}, 4-\mathrm{F}, \\
4-\mathrm{OCH}_{3}, 4-\mathrm{CH}_{3}, \\
3-\mathrm{NO}_{2}, 4-\mathrm{NO}_{2}\end{array}$ \\
\hline & $\sigma^{+}$ & 0.819 & 191.80 & 0.530 & 1.409 & 9 & $\begin{array}{c}\mathrm{H}, 4-\mathrm{Br}, 2-\mathrm{Cl}, 4-\mathrm{Cl}, 4-\mathrm{F}, \\
4-\mathrm{OCH}_{3}, 4-\mathrm{CH}_{3}, \\
3-\mathrm{NO}_{2}, 4-\mathrm{NO}_{2}\end{array}$ \\
\hline & $\sigma_{\mathrm{I}}$ & 0.904 & 191.04 & 2.151 & 1.312 & 8 & $\begin{array}{c}\mathrm{H}, 2-\mathrm{Cl}, 4-\mathrm{Cl}, 4-\mathrm{F}, 4- \\
\mathrm{OCH}_{3}, \\
4-\mathrm{CH}_{3}, 3-\mathrm{NO}_{2}, 4-\mathrm{NO}_{2}\end{array}$ \\
\hline$\bigcup_{\infty}$ & $\sigma_{\mathrm{R}}$ & 0.884 & 191.94 & 0.483 & 1.434 & 9 & $\begin{array}{c}\mathrm{H}, 4-\mathrm{Br}, 2-\mathrm{Cl}, 4-\mathrm{Cl}, 4-\mathrm{F}, \\
4-\mathrm{OCH}_{3}, 4-\mathrm{CH}_{3}, \\
3-\mathrm{NO}_{2}, 4-\mathrm{NO}_{2}\end{array}$ \\
\hline & $\mathrm{F}$ & 0.905 & 190.85 & 2.546 & 1.242 & 8 & $\begin{array}{c}\mathrm{H}, 2-\mathrm{Cl}, 4-\mathrm{Cl}, 4-\mathrm{F}, \\
4-\mathrm{OCH}_{3}, 4-\mathrm{CH}_{3}, \\
\text { 3- } \mathrm{NO}_{2}, 4-\mathrm{NO}_{2}\end{array}$ \\
\hline & $\mathrm{R}$ & 0.807 & 191.95 & 0.405 & 1.434 & 9 & $\begin{array}{c}\mathrm{H}, 4-\mathrm{Br}, 2-\mathrm{Cl}, 4-\mathrm{Cl}, 4-\mathrm{F}, \\
4-\mathrm{OCH}_{3}, 4-\mathrm{CH}_{3}, \\
3-\mathrm{NO}_{2}, 4-\mathrm{NO}_{2}\end{array}$ \\
\hline \multirow{3}{*}{$\bigcup_{\infty}^{\infty}$} & $\sigma$ & 0.853 & 125.34 & -1.841 & 0.958 & 9 & $\begin{array}{c}\mathrm{H}, 4-\mathrm{Br}, 2-\mathrm{Cl}, 4-\mathrm{Cl}, 4-\mathrm{F}, \\
4-\mathrm{OCH}_{3}, 4-\mathrm{CH}_{3}, \\
\text { 3- } \mathrm{NO}_{2}, 4-\mathrm{NO}_{2}\end{array}$ \\
\hline & $\sigma^{+}$ & 0.843 & 125.11 & -0.905 & 1.091 & 9 & $\begin{array}{c}\mathrm{H}, 4-\mathrm{Br}, 2-\mathrm{Cl}, 4-\mathrm{Cl}, 4-\mathrm{F}, \\
4-\mathrm{OCH}_{3}, 4-\mathrm{CH}_{3}, \\
3-\mathrm{NO}_{2}, 4-\mathrm{NO}_{2}\end{array}$ \\
\hline & $\sigma_{\mathrm{I}}$ & 0.838 & 125.62 & -1.672 & 1.104 & 9 & $\mathrm{H}, 4-\mathrm{Br}, 2-\mathrm{Cl}, 4-\mathrm{Cl}, 4-\mathrm{F}$, \\
\hline
\end{tabular}




\begin{tabular}{|c|c|c|c|c|c|c|c|}
\hline & & & & & & & $\begin{array}{c}4-\mathrm{OCH}_{3}, 4-\mathrm{CH}_{3}, \\
3-\mathrm{NO}_{2}, 4-\mathrm{NO}_{2}\end{array}$ \\
\hline & $\sigma_{\mathrm{R}}$ & 0.905 & 124.55 & -2.970 & 0.965 & 8 & $\begin{array}{l}\mathrm{H}, 4-\mathrm{Br}, 2-\mathrm{Cl}, 4-\mathrm{Cl}, 4-\mathrm{F}, \\
4-\mathrm{OCH}_{3}, 4-\mathrm{CH}_{3}, 4-\mathrm{NO}_{2}\end{array}$ \\
\hline & $\mathrm{F}$ & 0.835 & 125.57 & -1.478 & 1.119 & 9 & $\begin{array}{c}\mathrm{H}, 4-\mathrm{Br}, 2-\mathrm{Cl}, 4-\mathrm{Cl}, 4-\mathrm{F}, \\
4-\mathrm{OCH}_{3}, 4-\mathrm{CH}_{3}, \\
3-\mathrm{NO}_{2}, 4-\mathrm{NO}_{2}\end{array}$ \\
\hline & $\mathrm{R}$ & 0.905 & 124.56 & -2.151 & 1.033 & 8 & $\begin{array}{l}\mathrm{H}, 4-\mathrm{Br}, 2-\mathrm{Cl}, 4-\mathrm{Cl}, 4-\mathrm{F}, \\
4-\mathrm{OCH}_{3}, 4-\mathrm{CH}_{3}, 4-\mathrm{NO}_{2}\end{array}$ \\
\hline \multirow{6}{*}{$\bigcup_{0}^{\infty}$} & $\sigma$ & 0.902 & 144.63 & -3.976 & 1.050 & 8 & $\begin{array}{c}\mathrm{H}, 4-\mathrm{Br}, 4-\mathrm{Cl}, 4-\mathrm{F}, 4- \\
\mathrm{OCH}_{3}, \\
4-\mathrm{CH}_{3}, 3-\mathrm{NO}_{2}, 4-\mathrm{NO}_{2}\end{array}$ \\
\hline & $\sigma^{+}$ & 0.904 & 144.28 & -2.951 & 0.969 & 8 & $\begin{array}{c}\mathrm{H}, 4-\mathrm{Br}, 4-\mathrm{Cl}, 4-\mathrm{F}, 4- \\
\mathrm{OCH}_{3}, \\
4-\mathrm{CH}_{3}, 3-\mathrm{NO}_{2}, 4-\mathrm{NO}_{2}\end{array}$ \\
\hline & $\sigma_{\mathrm{I}}$ & 0.867 & 145.98 & -5.553 & 1.082 & 9 & $\begin{array}{c}\mathrm{H}, 4-\mathrm{Br}, 2-\mathrm{Cl}, 4-\mathrm{Cl}, \\
4-\mathrm{F}, 4-\mathrm{OCH}_{3}, 4-\mathrm{CH}_{3}, \\
\text { 3- } \mathrm{NO}_{2}, 4-\mathrm{NO}_{2}\end{array}$ \\
\hline & $\sigma_{\mathrm{R}}$ & 0.837 & 143.42 & -2.970 & 1.729 & 9 & $\begin{array}{c}\mathrm{H}, 4-\mathrm{Br}, 2-\mathrm{Cl}, 4-\mathrm{Cl}, 4-\mathrm{F}, \\
4-\mathrm{OCH}_{3}, 4-\mathrm{CH}_{3}, \\
3-\mathrm{NO}_{2}, 4-\mathrm{NO}_{2}\end{array}$ \\
\hline & $\mathrm{F}$ & 0.846 & 145.69 & -4.573 & 1.339 & 9 & $\begin{array}{c}\mathrm{H}, 4-\mathrm{Br}, 4-\mathrm{Cl}, 4-\mathrm{OCH}_{3}, \\
4-\mathrm{CH}_{3}, 3-\mathrm{NO}_{2}, 4-\mathrm{NO}_{2}\end{array}$ \\
\hline & $\mathrm{R}$ & 0.853 & 143.28 & -2.921 & 1.680 & 9 & $\begin{array}{c}\mathrm{H}, 4-\mathrm{Br}, 2-\mathrm{Cl}, 4-\mathrm{Cl}, 4-\mathrm{F}, \\
4-\mathrm{OCH}_{3}, 4-\mathrm{CH}_{3}, \\
3-\mathrm{NO}_{2}, 4-\mathrm{NO}_{2}\end{array}$ \\
\hline
\end{tabular}




\section{2. IR spectral study}

The synthesized chalcones in the present study are shown in Scheme 1. The carbonyl stretching frequencies $\left(\mathrm{cm}^{-1}\right)$ of $s$-cis and $s$-trans of isomers are tabulated in Table 2 and the corresponding conformers are shown in Fig. 2.
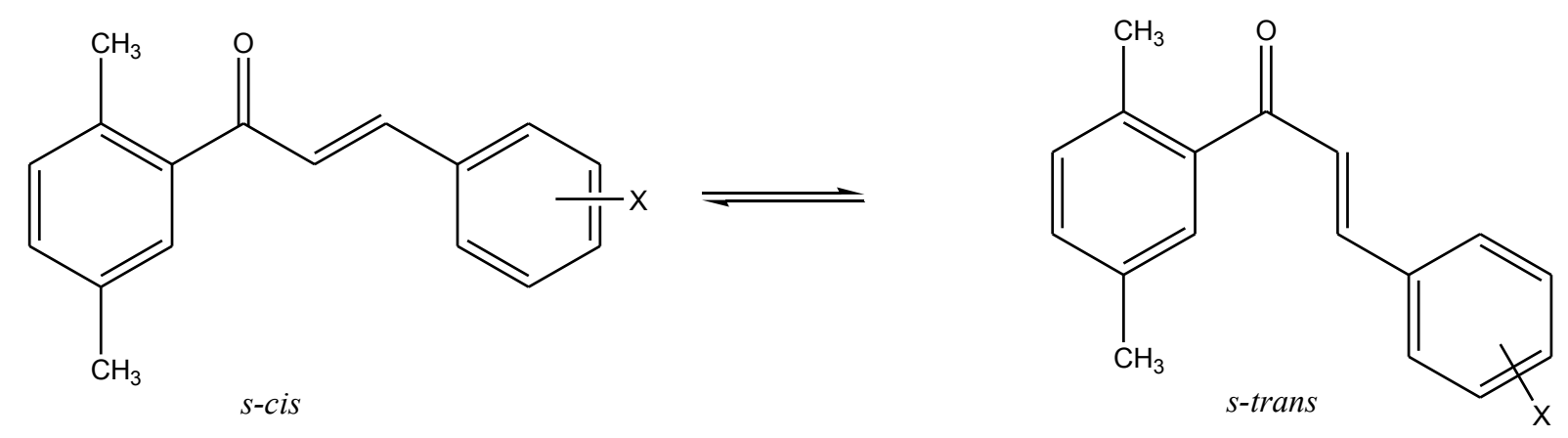

Fig. 2. The $s$-cis and $s$-trans conformers of 2',5'-dimethyl phenyl chalcones.

The assigned carbonyl stretching frequencies $\left(\mathrm{cm}^{-1}\right)$ of $s$-cis and $s$-trans isomers of present study are presented in Table 2 and the corresponding conformers are shown in Fig. 2. The stretching frequencies for carbonyl absorption are assigned based on the assignments made by Hays and Timmons [40] for s-cis and s-trans conformers at 1690 and $1670 \mathrm{~cm}^{-1}$, respectively. These data have been correlated with Hammett substituent constants and SwainLupton's constants [39] and are presented in Table 3. In this correlation the structure parameter Hammett equation employed is as shown in the following equation:

$$
v=\rho \sigma+v_{0}
$$

where $v$ is the carbonyl frequencies of substituted system and $v_{0}$ is the corresponding quantity of unsubstitued system; $\sigma$ is a Hammett substituent constant, which in principle is characteristics of the substituent and $\rho$ is a reaction constant which is depend upon the nature of the reaction.

From table 3, the results of single parameter statistical analysis of carbonyl frequencies of $s$-cis with all Hammett substituent constants and $F$ and $R$ parameters have shown poor correlations $(r<0.900)$. This failure in correlation is due to the incapability of polar, inductive, field and resonance effects of the substituents and associated with the resonance conjugative structure as shown in Fig. 1.

The infrared frequency of CO s-trans, except those with 3- $\mathrm{NO}_{2}$ and 4- $\mathrm{NO}_{2}$ substituents have shown satisfactory correlations with the Hammett substituent constants $\sigma(\mathrm{r}=0.905)$ and $\sigma^{+}(\mathrm{r}=0.904)$. The remaining Hammett substituent constants $\sigma_{\mathrm{I}}, \sigma_{\mathrm{R}}$ and $F$ and $R$ parameters have shown poor correlations $(\mathrm{r}<0.900)$. This is due to the weak inductive, field and resonance effects of the substituents and associated with the resonance conjugative structure as shown in Fig. 1.

The infrared frequency of $\mathrm{CH}_{i p}$, except those with $\mathrm{H}$ (parent) and 4- $\mathrm{NO}_{2}$ substituents have shown satisfactory correlation with Hammett substituent constant $\sigma(\mathrm{r}=0.905)$ and except those with $\mathrm{H}$ (parent), 3- $\mathrm{NO}_{2}$ and 4- $\mathrm{NO}_{2}$ substituents have shown satisfactory correlation with Hammett substituent constant $\sigma^{+}(\mathrm{r}=0.905)$ and except those with $3-\mathrm{NO}_{2}$ 
and 4-NO $\mathrm{NO}_{2}$ substituents have shown satisfactory correlation with Hammett substituent constant $\sigma_{\mathrm{I}}(\mathrm{r}=0.905)$. The remaining Hammett substituent constant $\sigma_{\mathrm{R}}$ and $F$ and $R$ parameters have shown poor correlations $(\mathrm{r}<0.900)$ This is attributed to the weak field and resonance effects of the substituents for predicting the reactivity on the infrared requency through resonance as per the conjugative structure as shown in Fig. 1.

The infrared frequency of $\mathrm{CH}_{o p}$ with all Hammett substituent constants and $F$ and $R$ parameters has shown poor correlations $(\mathrm{r}<0.900)$, and also the infrared frequency of $\mathrm{CH}=\mathrm{CH}_{o p}$ and $\mathrm{C}=\mathrm{C}_{o p}$ with all Hammett substituent constants and $F$ and $R$ parameters has shown poor correlations $(\mathrm{r}<0.900)$. This failure in correlation is due to the incapability of polar, inductive, field and resonance effects of the substituents and associated with the resonance conjugative structure as shown in Fig. 1

Some of the single parameter correlations with Hammett substituent constants were not obeyed in the regression. While seeking these parameters in multi-regression, with $\mathrm{F}$ and R Swain-Lupton's constants [39], they gave satisfactory correlations with the infrared red group frequencies. The multi correlation equations are given in $(5-16)$.

$$
\begin{aligned}
& v \mathrm{CO}_{\text {s-cis }}\left(\mathrm{cm}^{-1}\right)=1660.83( \pm 2.656)+3.561( \pm 5.329) \sigma_{\mathrm{I}}-0.025( \pm 6.142) \sigma_{\mathrm{R}} \\
& (R=0.926, \mathrm{n}=9, \mathrm{P}>90 \%) \\
& v \mathrm{CO}_{s-c i s}\left(\mathrm{~cm}^{-1}\right)=1660.98( \pm 2.603)+3.156( \pm 5.129) \mathrm{F}+0.217( \pm 5.219) \mathrm{R} \\
& (R=0.924, \mathrm{n}=9, \mathrm{P}>90 \%) \\
& \nu \mathrm{CO}_{\text {s-trans }}\left(\mathrm{cm}^{-1}\right)=1589.37( \pm 14.705)-33.008( \pm 29.498) \sigma_{\mathrm{I}}-51.234( \pm 33.999) \sigma_{\mathrm{R}} \\
& (R=0.963, \mathrm{n}=9, \mathrm{P}>95 \%) \\
& v \mathrm{CO}_{s-\text { trans }}\left(\mathrm{cm}^{-1}\right)=1589.53( \pm 14.541)-34.866( \pm 28.648) \mathrm{F}-4.535( \pm 29.153) \mathrm{R} \\
& (R=0.961, \mathrm{n}=9, \mathrm{P}>95 \%) \\
& v \mathrm{CH}_{i p}\left(\mathrm{~cm}^{-1}\right)=1161.86( \pm 3.356)+10.263( \pm 6.732) \sigma_{\mathrm{I}}+5.674( \pm 7.759) \sigma_{\mathrm{R}} \\
& (R=0.958, \mathrm{n}=9, \mathrm{P}>95 \%) \\
& v \mathrm{CH}_{i p}\left(\mathrm{~cm}^{-1}\right)=1162.66( \pm 3.357)+8.935( \pm 6.615) \mathrm{F}+6.598( \pm 6.731) \mathrm{R} \\
& (R=0.955, \mathrm{n}=9, \mathrm{P}>95 \%) \\
& v \mathrm{CH}_{o p}\left(\mathrm{~cm}^{-1}\right)=760.437( \pm 17.097)+73.135( \pm 34.295) \sigma_{\mathrm{I}}-16.690( \pm 39.529) \sigma_{\mathrm{R}} \\
& (R=0.965, \mathrm{n}=9, \mathrm{P}>95 \%) \\
& v \mathrm{CH}_{o p}\left(\mathrm{~cm}^{-1}\right)=759.282( \pm 15.678)+74.367( \pm 30.887) \mathrm{F}-9.158( \pm 31.432) \mathrm{R} \\
& (R=0.970, \mathrm{n}=9, \mathrm{P}>95 \%) \\
& v \mathrm{CH}=\mathrm{CH}_{o p}\left(\mathrm{~cm}^{-1}\right)=1014.82( \pm 2.911)-2.106( \pm 5.839) \sigma_{\mathrm{I}}-2.550( \pm 6.730) \sigma_{\mathrm{R}} \\
& (R=0.922, \mathrm{n}=9, \mathrm{P}>90 \%) \\
& v \mathrm{CH}=\mathrm{CH}_{o p}\left(\mathrm{~cm}^{-1}\right)=1015.31( \pm 2.792)-3.362( \pm 5.502) \mathrm{F}-2.178( \pm 5.599) \mathrm{R} \\
& (R=0.927, \mathrm{n}=9, \mathrm{P}>90 \%) \\
& v \mathrm{C}=\mathrm{C}_{o p}\left(\mathrm{~cm}^{-1}\right)=559.400( \pm 26.698)-63.259( \pm 53.554) \sigma_{\mathrm{I}}+64.892( \pm 61.726) \sigma_{\mathrm{R}} \\
& (R=0.952, \mathrm{n}=9, \mathrm{P}>95 \%) \\
& v \mathrm{C}=\mathrm{C}_{o p}\left(\mathrm{~cm}^{-1}\right)=558.746( \pm 25.738)-56.340( \pm 50.705) \mathrm{F}+53.963( \pm 51.599) \mathrm{R} \\
& (R=0.953, \mathrm{n}=9, \mathrm{P}>95 \%)
\end{aligned}
$$




\section{3. NMR Spectral study}

\section{3. 1. ${ }^{1} \mathrm{H}$ NMR spectra}

The ${ }^{1} \mathrm{H}$ NMR spectra of synthesized chalcones have been recorded using deuteriochloroform employing tetramethylsilane (TMS) as internal standard. The ethylenic protons signals of the chalcones were assigned from their spectra. They were calculated as $\mathrm{AB}$ or $\mathrm{AA}^{\prime}$ or $\mathrm{BB}^{\prime}$ systems respectively. The lower chemical shifts (ppm) obtained for $\mathrm{H} \alpha$ and higher chemical shifts (ppm) obtained for $\mathrm{H}_{\beta}$ in this series of ketones. The vinyl protons give an $\mathrm{AB}$ pattern and the $\beta$-proton doublets were well separated from the signals of the aromatic protons. The assigned vinyl proton chemical shifts $\delta(\mathrm{ppm})$ of all ketones were presented in Table 2. In nuclear magnetic resonance spectra, the proton or the ${ }^{13} \mathrm{C}$ chemical shifts $(\delta)$ depends on the electronic environment of the nuclei concerned. The assigned vinyl proton chemical shifts (ppm) have been correlated with reactivity parameters using Hammett equation in the form of

$$
\log \delta=\log \delta_{0}+\rho \sigma
$$

where $\delta_{0}$ is the chemical shift of unsubstitued ketones. The assigned $\mathrm{H} \alpha$ and $\mathrm{H}_{\beta}$ proton chemical shifts (ppm) are correlated with various Hammett sigma constants. The results of statistical analysis [1,4,31-38] are presented in Table 3 .

The $\mathrm{H} \alpha$ chemical shifts $(\delta$, ppm) correlated with Hammett constants and $\mathrm{F}$ and $\mathrm{R}$ parameters, except those with $\mathrm{H}$ (parent) and $4-\mathrm{CH}_{3}$ have shown satisfactory correlations with a few Hammett substituent constants $\sigma(r=0.917)$ and $\sigma_{I}(r=0.907)$, and except those with 4$\mathrm{Br}$ and 2- $\mathrm{Cl}$ have shown satisfactory correlations with Hammett substituent constant $\sigma^{+}(\mathrm{r}=$ 0.907), and except that with 2-F substituent have shown satisfactory correlation with Hammett substituent constant $\sigma_{\mathrm{R}}(\mathrm{r}=0.906)$ and $R$ parameter $(\mathrm{r}=0.920)$. The remaining $\mathrm{F}$ parameter failure in correlation for both chemical shifts are the reasons stated earlier and associated with the resonance - conjugated structure shown in Fig. 1. The $\mathrm{H}_{\beta}$ chemical shifts $(\delta, \mathrm{ppm})$ correlated with Hammett constants and $\mathrm{F}$ and $\mathrm{R}$ parameters, except those with $\mathrm{H}$ (parent) and 4- $\mathrm{CH}_{3}$ substituents have shown satisfactory correlation with Hammett substituent constant $\sigma_{\mathrm{I}}(\mathrm{r}=0.905)$ and except those with $\mathrm{H}$ (parent) and 4-F substituents have shown satisfactory correlation with $\mathrm{F}$ parameter $(\mathrm{r}=0.905)$. The remaining Hammett substituent constants $\sigma, \sigma^{+}, \sigma_{\mathrm{R}}$ and $R$ parameter have shown poor correlations $(\mathrm{r}<0.900)$. This is attributed to the weak polar and resonance effects of the substituents for predicting the reactivity on the chemical shifts through resonance as per the conjugative structure shown in Fig. 1. Some of the single parameter correlations with Hammett substituent constants were not obeyed in the regression. While seeking these parameters in multi-regression, with $\mathrm{F}$ and R Swain-Lupton's [39] constants, they gave satisfactory correlations with the chemical shifts of $(\delta, \mathrm{ppm}) \mathrm{H} \alpha$ and $\mathrm{H} \beta$. The multi correlation equations are given in (18-21).

$$
\begin{gathered}
\delta \mathrm{H}_{\alpha}(\mathrm{ppm})=7.152( \pm 0.022)+0.128( \pm 0.045) \sigma_{\mathrm{I}}+0.324( \pm 0.052) \sigma_{\mathrm{R}} \\
(R=0.945, \mathrm{n}=9, \mathrm{P}>90 \%) \\
\delta \mathrm{H}_{\alpha}(\mathrm{ppm})=7.153( \pm 0.018)+0.144( \pm 0.035) \mathrm{F}+0.292( \pm 0.036) \mathrm{R} \\
(R=0.966, \mathrm{n}=9, \mathrm{P}>95 \%) \\
\delta \mathrm{H}_{\beta}(\mathrm{ppm})=7.373( \pm 0.067)+0.226( \pm 0.135) \sigma_{\mathrm{I}}+0.143( \pm 0.156) \sigma_{\mathrm{R}} \\
(R=0.963, \mathrm{n}=9, \mathrm{P}>95 \%) \\
\delta \mathrm{H}_{\beta}(\mathrm{ppm})=7.376( \pm 0.065)+0.226( \pm 0.128) \mathrm{F}+0.147( \pm 0.130) \mathrm{R} \\
(R=0.964, \mathrm{n}=9, \mathrm{P}>95 \%)
\end{gathered}
$$




\section{3. 2. ${ }^{13} \mathrm{C}$ NMR spectra}

Scientists and physical organic chemists [1,4,22-23,31-38,41-44], have made extensive study of ${ }^{13} \mathrm{C}$ NMR spectra for a large number of different ketones and styrenes. The assigned vinyl $\mathrm{C}_{\alpha}, \mathrm{C}_{\beta}$ and carbonyl carbon chemical shifts are presented in Table 2 . The results of statistical analysis are given in Table 3. The $\mathrm{C} \alpha$ chemical shifts, except that with $3-\mathrm{NO}_{2}$ substituent have shown satisfactory correlation with Hammett substituent constant $\sigma_{R}(r=$ $0.905)$ and $\mathrm{R}$ parameter $(\mathrm{r}=0.905)$. The remaining Hammett substituent constants $\sigma, \sigma^{+} \& \sigma_{\mathrm{I}}$ and $F$ parameter have shown poor correlations. This is attributed to the weak polar, inductive and field effects of the substituents for predicting the reactivity on the $\mathrm{C} \alpha$ chemical shifts through resonance as per the conjugative structure shown in Fig. 1 . The $\delta C_{\beta}$ chemical shift, except that with 2-Cl substituent have shown satisfactory correlations with a few Hammett substituent constants namely $\sigma(\mathrm{r}=0.902)$ and $\sigma^{+}(\mathrm{r}=0.904)$. The remaining Hammett substituent constant $\sigma_{\mathrm{I}}, \sigma_{\mathrm{R}}$ and $F$ and $\mathrm{R}$ parameters have shown poor correlations $(\mathrm{r}<0.900)$. This is due to the weak polar effect of the substituents for predicting the reactivity on the chemical shift through resonance as per the conjugative structure shown in Fig. 1. In carbonyl carbon chemical shifts, except that with 4-Br substituent have shown satisfactory correlations with Hammett substituent constant namely $\sigma_{\mathrm{I}}(\mathrm{r}=0.904)$ and $F$ parameter $(\mathrm{r}=0.905)$. The remaining Hammett substituent constants $\sigma, \sigma^{+}, \sigma_{\mathrm{R}}$ and $R$ parameter have shown poor correlations. This is due to the reasons stated in earlier and associated with the resonance conjugative structure shown in Fig. 1. Some of the single parameter correlation were fails in regression, while seeking the multi-regression analysis through Swain Lupton's [39] parameter correlations were satisfactorily obtained within these carbon chemical shifts and the regression equations are given in 22-27.

$$
\begin{gathered}
\delta \mathrm{CO}(\mathrm{ppm})=191.085( \pm 0.979)+2.131( \pm 1.965) \sigma_{\mathrm{I}}+0.196( \pm 2.265) \sigma_{\mathrm{R}} \\
(R=0.941, \mathrm{n}=9, \mathrm{P}>90 \%) \\
\delta \mathrm{CO}(\mathrm{ppm})=190.940( \pm 0.898)+2.564( \pm 1.770) \mathrm{F}+0.503( \pm 1.801) \mathrm{R} \\
(R=0.951, \mathrm{n}=9, \mathrm{P}>95 \%) \\
\delta \mathrm{C}_{\alpha}(\mathrm{ppm})=125.115( \pm 0.663)-1.389( \pm 1.330) \sigma_{\mathrm{I}}-2.783( \pm 1.533) \sigma_{\mathrm{R}} \\
(R=0.967, \mathrm{n}=9, \mathrm{P}>95 \%) \\
\delta \mathrm{C}_{\alpha}(\mathrm{ppm})=125.184( \pm 0.6796)-1.559( \pm 1.339) \mathrm{F}-2.210( \pm 1.362) \mathrm{R} \\
(R=0.962, \mathrm{n}=9, \mathrm{P}>95 \%) \\
\delta \mathrm{C}_{\beta}(\mathrm{ppm})=145.576( \pm 0.704)-5.325( \pm 1.412) \sigma_{\mathrm{I}}-2.253( \pm 1.628) \sigma_{\mathrm{R}} \\
(R=0.986, \mathrm{n}=9, \mathrm{P}>95 \%) \\
\delta \mathrm{C}_{\beta}(\mathrm{ppm})=145.144( \pm 0.743)-4.687( \pm 1.464) \mathrm{F}-3.099( \pm 1.490) \mathrm{R} \\
(R=0.983, \mathrm{n}=9, \mathrm{P}>95 \%)
\end{gathered}
$$

\section{4. Antimicrobial activities}

\section{4. 1. Antibacterial sensitivity assay}

Antibacterial sensitivity assay of all chalcones were performed using Kirby-Bauer [29] disc diffusion technique. In each Petri plate about $0.5 \mathrm{~mL}$ of the test bacterial sample was spread uniformly over the solidified Mueller Hinton agar using sterile glass spreader. Then the discs with $5 \mathrm{~mm}$ 


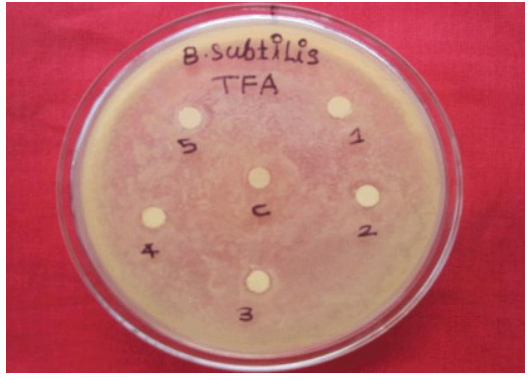

Plate-1

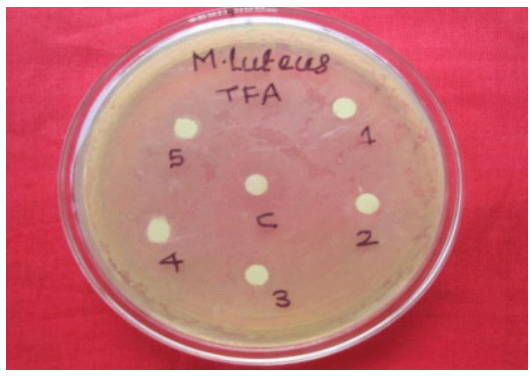

Plate-3

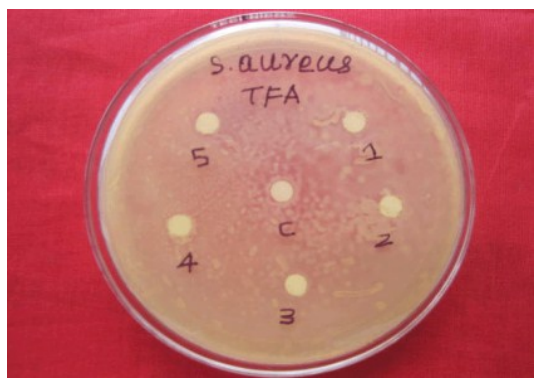

Plate-5

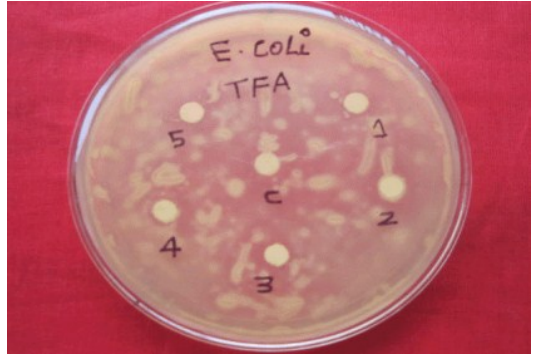

Plate-7

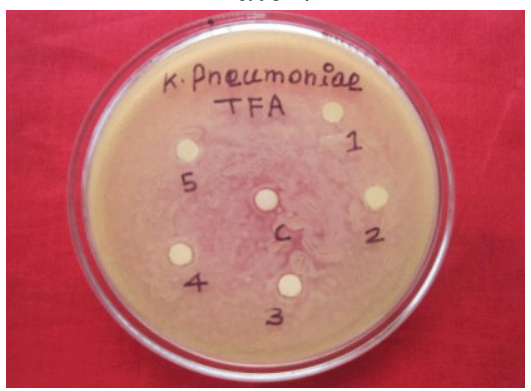

Plate-9

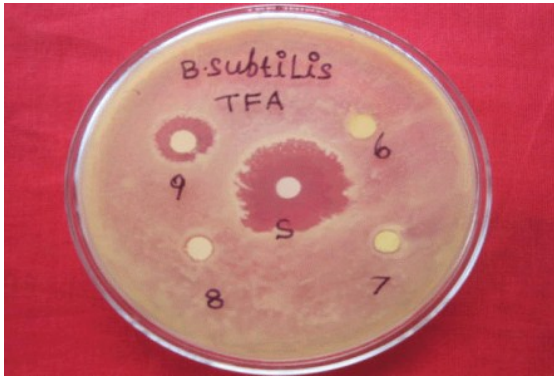

Plate-2

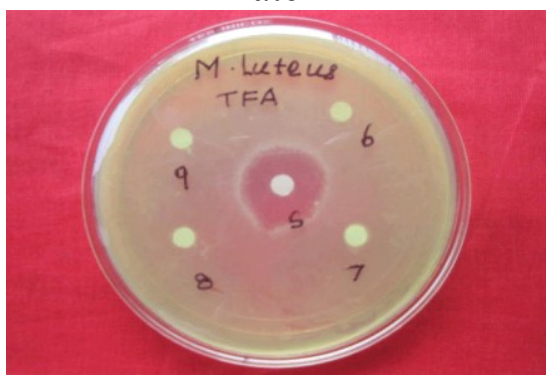

Plate-4

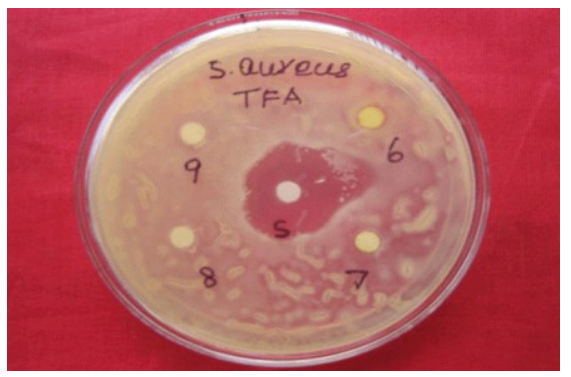

Plate-6

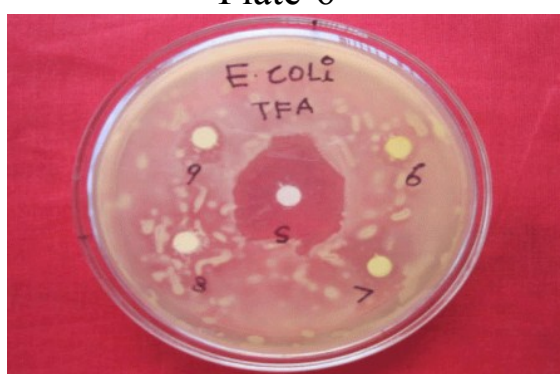

Plate-8

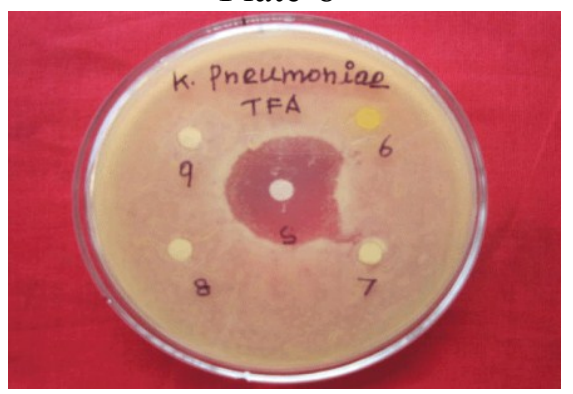

Plate-10

Fig. 3. Antibacterial activities of substituted 2,5-dimethyl phenyl chalcone. 
diameter made up of Whatmann No.1 filter paper, impregnated with the solution of the compound was placed on the medium using sterile foreceps. The plates were incubated for 24 hours at $37^{\circ} \mathrm{C}$ by keeping the plates upside down to prevent the collection of water droplets over the medium. After 24 hours, the plates were visually examined and the diameter values of the zone of inhibition were measured. Triplicate results were recorded by repeating the same procedure.

The antibacterial effect of the styryl 2,5-dimethyl phenyl chalcones is shown in Fig. 3 for Plates (1)-(10). Analysis of the zone of inhibition as given Table 4 and the Clustered column Chart Fig. 4, 4- $\mathrm{NO}_{2}$ Only one substituent has shown excellent antibacterial activity of B.subtilis species. All the species have shown moderate antibacterial activity of some substituents 4-Cl, 4-F and 4- $\mathrm{CH}_{3}$. All the substituents except 4-Br and 3- $\mathrm{NO}_{2}$ have shown moderate antibacterial activity of E.coli species.

Table 4. Antibacterial activity of substituted styryl 2,5-dimethyl phenyl chalcones.

\begin{tabular}{|c|c|c|c|c|c|c|}
\hline \multirow{3}{*}{ Entry } & \multirow{3}{*}{ Substt. } & \multicolumn{5}{|c|}{ Zone of Inhibition (mm) } \\
\hline & & \multicolumn{3}{|c|}{ Gram positive Bacteria } & \multicolumn{2}{|c|}{ Gram negative Bacteria } \\
\hline & & B. subtilis & M. luteus & S. aureus & E. coli & K. pneumoniae \\
\hline 1 & $\mathrm{H}$ & - & 6 & 6 & 6 & - \\
\hline 2 & $4-\mathrm{Br}$ & 7 & - & - & - & 6 \\
\hline 3 & $2-\mathrm{Cl}$ & 6 & - & - & 8 & 6 \\
\hline 4 & $4-\mathrm{Cl}$ & 6 & 7 & 7 & 7 & 6 \\
\hline 5 & $4-F$ & 6 & 6 & 6 & 6 & 6 \\
\hline 6 & $4-\mathrm{OCH}_{3}$ & - & - & 7 & 8 & - \\
\hline 7 & $4-\mathrm{CH}_{3}$ & 6 & 6 & 6 & 6 & 6 \\
\hline 8 & $3-\mathrm{NO}_{2}$ & 6 & 6 & - & - & - \\
\hline 9 & $4-\mathrm{NO}_{2}$ & 10 & - & 8 & 8 & 6 \\
\hline Standard & Ampicillin & 15 & 14 & 15 & 16 & 16 \\
\hline Control & DMSO & - & - & - & - & - \\
\hline
\end{tabular}




\section{Antibacterial activity of substituted}

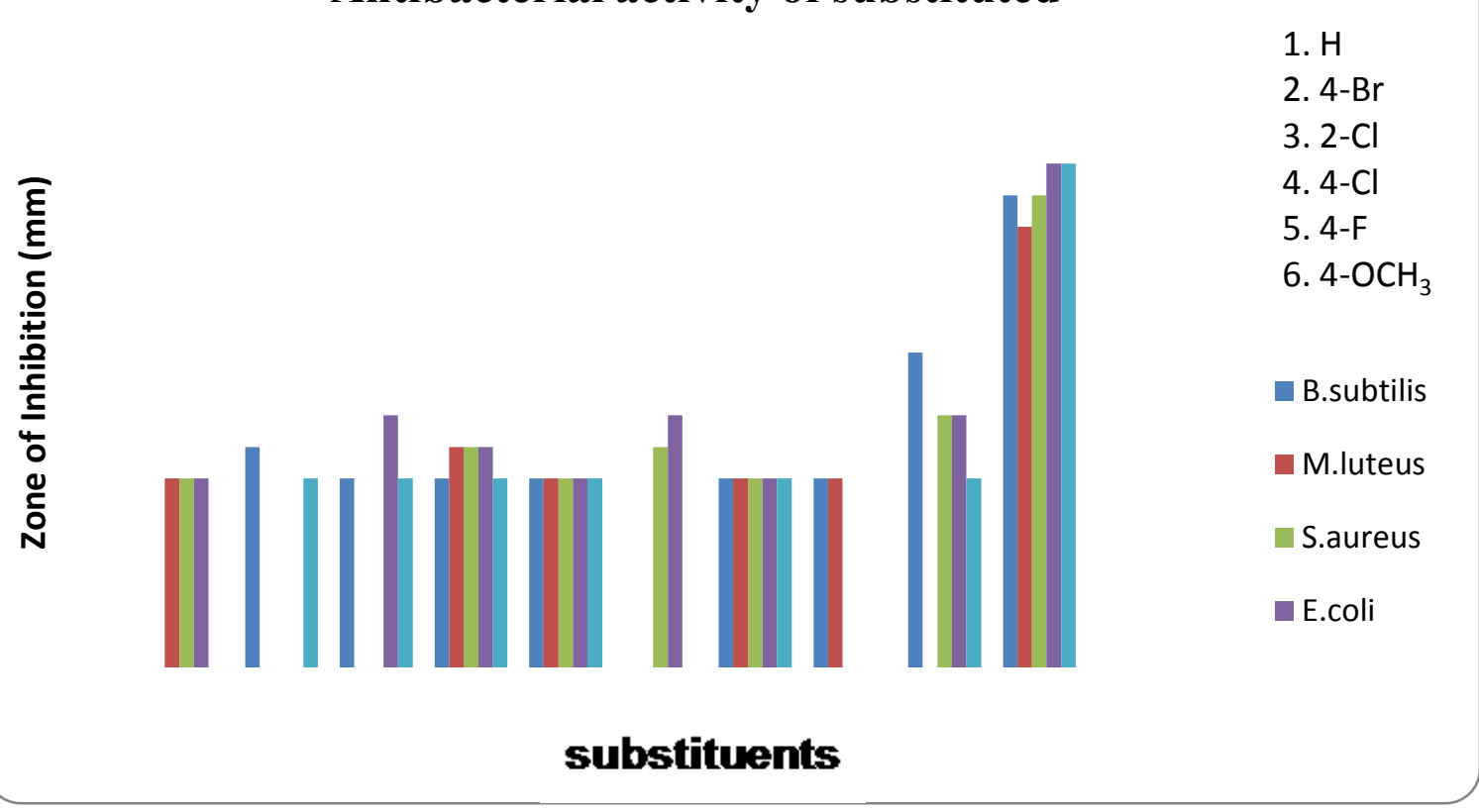

Fig. 4. Antibacterial activity of substituted styryl 2,5-dimethyl phenyl chalcone.

The substituent 4-Cl has shown equal activities against all bacteria except $B$. subtilis and $K$. pneumoniae and $4-\mathrm{F}$ and $4-\mathrm{CH}_{3}$ also has shown equal activities against all bacteria species. Only two substituent with $\mathrm{H}$ (parent) and $4-\mathrm{OCH}_{3}$ have shown poor activity of $B$. subtilis bacterial species. The substituents with 2-Cl, 4-Cl, 4-F, 4- $\mathrm{CH}_{3}$ and $3-\mathrm{NO}_{2}$ have shown equal activities against $B$. subtilis bacterial species and also substituents with $\mathrm{H}$ (parent), 4-F, 4- $\mathrm{CH}_{3}$ and 3- $\mathrm{NO}_{2}$ have shown equal activities against $M$. luteus bacterial species.

\section{4. 2. Antifungal sensitivity assay}

Antifungal sensitivity assay was performed using Kirby-Bauer [29] disc diffusion technique. PDA medium was prepared and sterilized as above. It was poured (ear bearing heating condition) in the Petri-plate which was already filled with $1 \mathrm{ml}$ of the fungal species. The plate was rotated clockwise and counter clock-wise for uniform spreading of the species. The discs were impregnated with the test solution. The test solution was prepared by dissolving $15 \mathrm{mg}$ of the Chalcone in $1 \mathrm{~mL}$ of DMSO solvent. The medium was allowed to solidify and kept for 24 hours. Then the plates were visually examined and the diameter values of zone of inhibition were measured. Triplicate results were recorded by repeating the same procedure. The antifungal effect of the styryl 2,5-dimethyl phenyl chalcones is shown in Fig. 5 for Plates (1-4). Analysis of the zone of inhibition as given Table 5 and the Clustered column Chart Fig. 6. Analysis of the Zone of inhibition $(\mathrm{mm})$ values reveals that only five substituent compounds with $\mathrm{H}$ (parent), 2-Cl, 4-F, 4- $\mathrm{OCH}_{3}$ and $3-\mathrm{NO}_{2}$ have shown moderate antifungal activities. The remaining four substituent compounds with $4-\mathrm{Br}, 4-\mathrm{Cl}, 4-\mathrm{CH}_{3}$ and $4-\mathrm{NO}_{2}$ have shown poor antifungal activities of fungal species namely A. niger. Analysis of the Zone of inhibition (mm) values reveals that only six substituent compounds with $\mathrm{H}$ (parent), 4- $\mathrm{Br}, 2-\mathrm{Cl}, 4-\mathrm{F}, 4-\mathrm{OCH}_{3}$ and 3$\mathrm{NO}_{2}$ have shown moderate antifungal activities. The remaining three substituent compounds with 4-Cl, 4- $\mathrm{CH}_{3}$ and $4-\mathrm{NO}_{2}$ have shown poor antifungal activities of fungal species namely T. viride. 


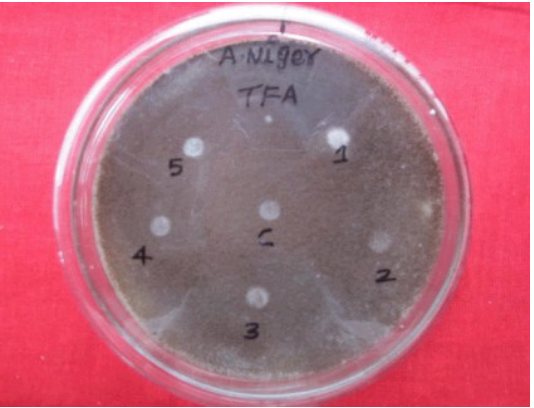

Plate-1

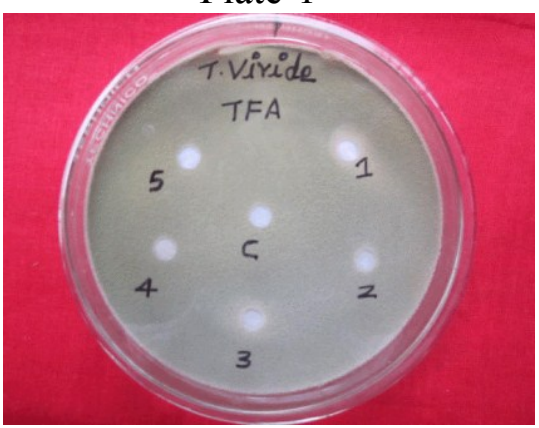

Plate-3

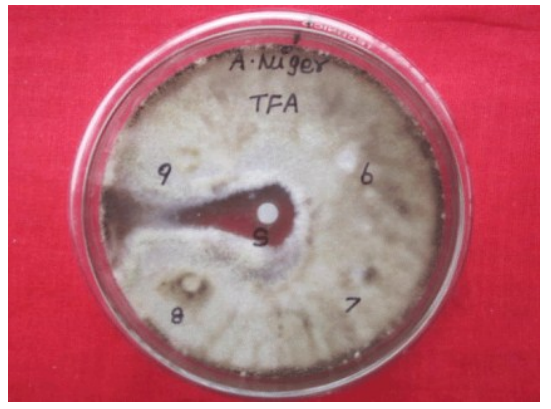

Plate-2

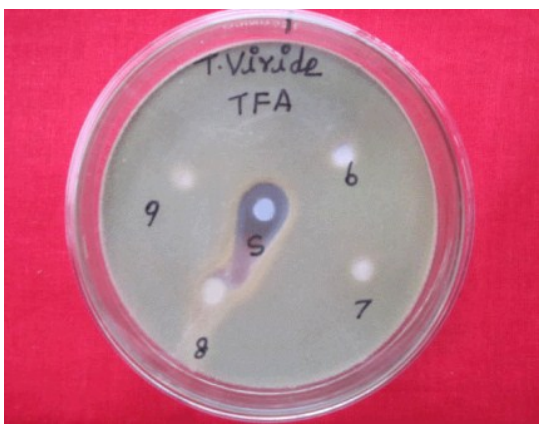

Plate-4

Fig. 5. Antifungal activities substituted styryl 2,5-dimethyl phenyl chalcones.

Table 5. Antifungal activity of substituted styryl 2,5-dimethyl phenyl chalcones.

\begin{tabular}{|c|c|c|c|}
\hline \multirow{2}{*}{ Entry } & \multirow{2}{*}{ Substt. } & \multicolumn{2}{|c|}{ Zone of Inhibition (mm) } \\
\cline { 3 - 4 } & & A. niger & T. viride \\
\hline 1 & $\mathrm{H}$ & 6 & 6 \\
\hline 2 & $4-\mathrm{Br}$ & - & 6 \\
\hline 3 & $2-\mathrm{Cl}$ & 6 & 6 \\
\hline 4 & $4-\mathrm{Cl}$ & - & - \\
\hline 5 & $4-\mathrm{F}$ & 6 & 6 \\
\hline 6 & $4-\mathrm{OCH}_{3}$ & 6 & 6 \\
\hline 7 & $4-\mathrm{CH}_{3}$ & - & - \\
\hline 8 & $3-\mathrm{NO}_{2}$ & 7 & 7 \\
\hline 9 & $4-\mathrm{NO}_{2}$ & - & - \\
\hline Standard & Miconazole & 11 & 10 \\
\hline control & DMSO & - & - \\
\hline
\end{tabular}




\section{Antifungal activityof substituted styryl 2,5-dimethyl phenyl ketone}

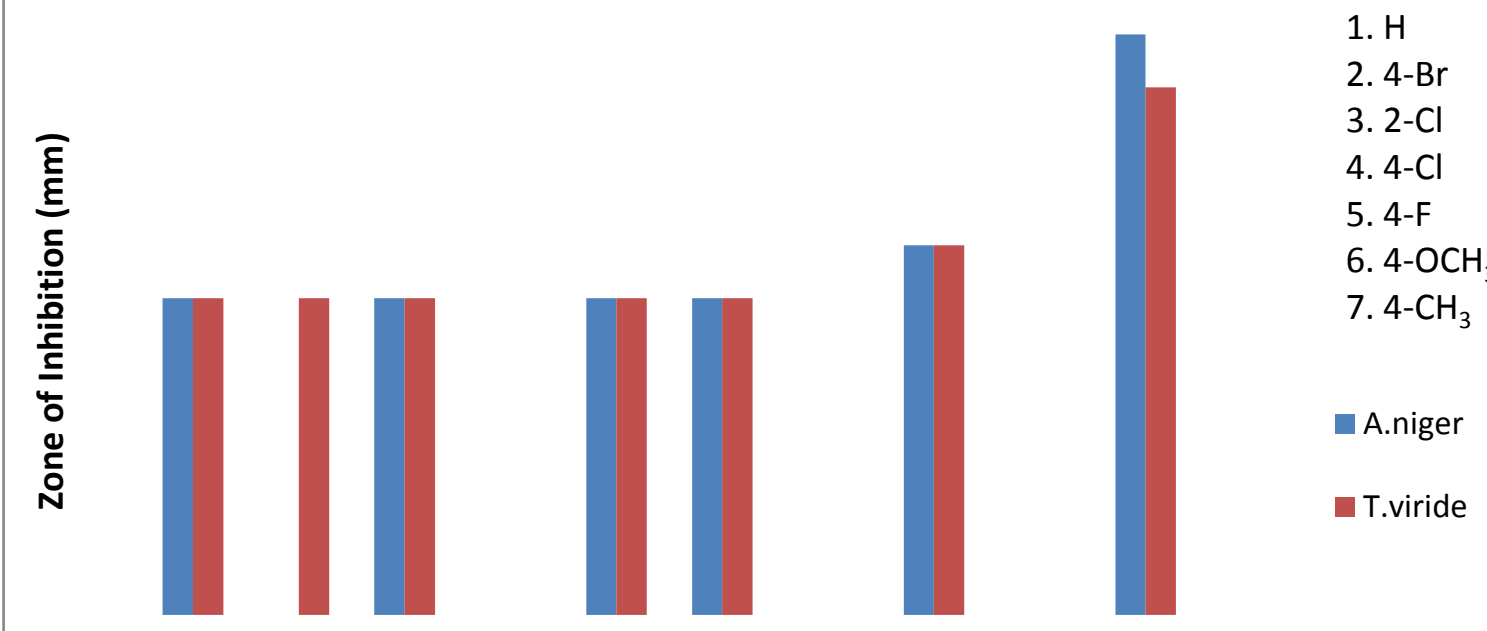

substituents

Fig. 6. Antifungal activity of substituted styryl 2,5-dimethyl phenyl chalcones-clustered column chart.

\section{CONCLUSIONS}

Some 2',5'-dimethyl phenyl chalcones have been synthesized by Crossed-Aldol condensation of 2,5-dimethyl acetophenone and substituted benzaldehydes in the presence sodium hydroxide and ethanol. This reaction protocol offers a simple, easier work-up procedure and good yields. The chalcones have been characterized by their physical constants, spectral data. The UV, IR, NMR spectral data of these chalcones has been correlated with Hammett substituent constants, F and R parameters. From the results of statistical analyses the effects of substituent on the spectral data have been studied. The antimicrobial activities of all synthesized chalcone have been studied using Bauer-Kirby method.

\section{Acknowledgement}

The authors wished to thank DST NMR Facility, SAIF, IIT, Madras, for recording NMR spectra of all compounds. 


\section{References}

[1] (a). Vanangamudi G, Ranganathan K, Thirunarayanan G, World J. Chem., 7(1), (2012) 22-33. (b). Arulkumaran R., Vijayakumar S., Sakthinathan S. P., Kamalakkannan D., Ranganathan K., Suresh R., Sundararajan R., Vanangamudi G., Thirunarayanan G., J. Chil. Chem. Soc. 58(2) (2013) 1553-1559.

[2] Yankep E., Fomumand Z. T., Dangne E., Phytochem. 46 (1997) 591-593.

[3] Thirunarayanan G., Vanangamudi G., E-J. Chem. 4 (1) (2007) 90-97.

[4] Ranganathan K., Arulkumaran R., Kamalakkannan D., Sundararajan R., Sakthinathan S. P., Vijayakumar S., Suresh R., Vanangamudi G., Thirumurthy K., Mayavel P., Thirunrayanan G., Int. J. Pharm. Med. and Bio. SC 1(1) (2012) 62-85.

[5] Venkat Reddy G, Maitraie G, Narsaiah D, Rambahu B, Rao R, Synth. Commun. 31(18) (2001) 2881-2884.

[6] Mulliken R. S., J. Chem. Phys. 7 (1939) 121-131.

[7] Hsieh H. K., Tsao L. T., Wang J. P., J. Pharm. Pharmacol. 52(2) (2000) 163-171.

[8] Viana G. S., Bandeira M. A., Matos F., J. Phytomed. 10 (2003) 189-195.

[9] Zhao L. M., Jin H. S., Sun L. P., Piao H. R., Quan Z. S., Bioorg. Med. Chem. Lett. 15(22) (2005) 5027-5029.

[10] Mukarami S., Muramatsu M., Aihara H., Otomo S., Biochem. Pharmacol. 42(7) (1991) 1447-1451.

[11] Liu M., Wilairat P., Go L. M., J. Med. Chem. 44(5) (2001) 4443-4452.

[12] Francesco E., Salvatore G., Luigi M., Phytochem. 68(7) (2007) 939-953.

[13] Onyilagna J. C., Malhotra B., Elder M., Towers G. H. N., Can. J. Plant. Pathol. 19 (1997) 133-137.

[14] Nielsen S. F., Chen M., Theander T. G., Kharazmi A., Bioorg. Med. Chem. Lett. 5 (1995) 449-452.

[15] Miranda C. L., Aponso G. L. M., J. Agricul Food Chem. 48 (2000) 3876-3884.

[16] Siva Kumar P. M., Geetha Babu S. K., Mukesh D, Chem. Pharm. Bull. 55(1) (2007) 4449.

[17] Satyanarayana M., Tiwari P., Tripathi K., Srivastava A. K., Pratap R., Bioorg. Med. Chem. Lett. 12 (2004) 883-889.

[18] Barford L., Kemp K., Hansen M., Kharazmi A., Inter Immunopharmacol. 2 (2007) 545.

[19] Maria J., Moa G., Mandado M., Chem. Phy. Lett. 446 (2007) 1-7.

[20] Anto R. J., Sukumaran K., Kuttan G., Rao M. A., Cancer Lett. 97 (1995) 33.

[21] Utpal B., Sahu A., Ali S. S., Kasoju L., Singh A., Food Res. Inter. 41 (2008) 1-15.

[22] Subramanian M., Vanangamudi G., Thirunarayanan G., Spectrochim. Acta 110A (2013) 116-123.

[23] Vanangamudi G., Subramanian M., Thirunarayanan G., Arabian J. Chem., 2013. DOI: 10.1016/j.arabjc.2013.03.006.

[24] Thirunarayanan G., J. Indian Chem. Soc. 84 (2008) 447-451. 
[25] Thirunarayanan G., Surya S., Srinivasan S., Vanangamudi G., Sathiyendiran V., Spectrochim. Acta. 75A (2010) 152-156.

[26] Liu X., Go M. L., Bioorg. Med.Chem. 14(1) (2006) 153-163.

[27] Arulkumaran R., Sundararajan R., Vanangamudi G., Subramanian M., Ravi K., Sathiyendidran V., Srinivasan S., Thirunarayanan G., IUP J. Chem. 3(1) (2010) 82-98.

[28] Deng J., Sanchez T., Lalith Q. A. M.. Bioorg. Med. Chem. 15(14) (2007) 4985-5002.

[29] Bauer A. W., Kirby W. M. M., Sherris J. C., Truck M., Am. J. Clin. Pathol. 45 (1966) 493-496.

[30] G. Thirunarayanan, Proceeding of the $46^{\text {th }}$ Annual Convention of Chemists and International Conf. Recent. Res. Trend. Chem. Sci. organised by by Ind. Chem. Soc., 2009 Dec. 2-6 Hosted by Vellore, Tamilnadu, Abst. 13, C13, 2009.

[31] Sathiyamoorthi K., Mala V., Suresh R., Sakthinathan S. P., Kamalakkannan D., Ranganathan K., Arulkumaran R., Sundararajan R., Vijayakumar S., Vanangamudi G., Thirunarayanan G., International Letters of Chemistry, Physics and Astronomy 7(2) (2013) 102-119.

[32] Sathiyamoorthi K., Mala V., Suresh R., Sakthinathan S. P., Kamalakkannan D., Vanangamudi G., Thirunarayanan G., Spectrochim Acta, 2013.

DOI: http://dx.doi.org/10.1016/j.saa.2013.04.048.

[33] Mala V., Sathiyamoorthy K., Sakthinathan S. P., Kamalakkannan D., Suresh R., Vanangamudi G., Thirunarayanan G., Q-Science Connect. 2013.

DOI: http://dx.doi.org/10.5339/connect.2013.

[34] Sekar K. G., Thirunarayanan G., International Letters of Chemistry, Physics and Astronomy 8(3) (2013) 249-258.

[35] Ranganathan K., Suresh R., Kamalakkannan D., Arulkumaran R., Sundararajan R., Sakthinathan S. P., Vijayakumar S., Vanangamudi G., Thirumurthy K., Mayavel P., Thirunarayanan G., International Letters of Chemistry, Physics and Astronomy 4 (2012) 66-75.

[36] Arulkumaran R., Vijayakumar S., Sundararajan R., Sakthinathan S. P., Kamalakkannan D., Suresh R., Ranganathan K., Rajakumar P. R., Vanangamudi G., Thirunarayanan G., International Letters of Chemistry, Physics and Astronomy 5 (2013) 21-38.

[37] Sakthinathan S. P., Suresh R., Mala V., Sathiyamoorthi K., Kamalakkannan D.,

Ranganathan K., Arulkumaran R., Vijayakumar S., Sundararajan R., Vanangamudi G., Thirunarayanan G., International Letters of Chemistry, Physics and Astronomy 6 (2013) 77-90.

[38] Sekar K. G., Thirunarayanan G., International Letters of Chemistry, Physics and Astronomy 8(2) (2013) 160-174.

[39] Swain C. G., Lupton E. C. Jr., J. Am. Chem. Soc. 90 (1968) 4328-4337.

[40] Hays W. P., Timmons C. J., Spectrochim. Acta. 24A (1968) 323-334.

[41] S. Vijayakumar, R. Arulkumaran, R. Sundararajan, S. P. Sakthinathan, R. Suresh, D. Kamalakkannan, K. Ranganathan, K. Sathiyamoorthy, V. Mala, G. Vanangamudi, G. Thirunarayanan, International Letters of Chemistry, Physics and Astronomy 9(1) (2013) 68-86. 
[42] Thirunarayanan G., Sekar K. G., International Letters of Chemistry, Physics and Astronomy 10 (2013) 18-34.

[43] R. Sundararajan, R. Arulkumaran, S. Vijayakumar, D. Kamalakkannan, R. Suresh, S. John Joseph, K. Ranganathan, S. P. Sakthinathan, G. Vanangamudi, G. Thirunarayanan, International Letters of Chemistry, Physics and Astronomy 1 (2014) 67-73.

[44] John Joseph S., Arulkumaran R., Kamalakkannan D., Sakthinathan S.P., Sundararajan R., Suresh R., Vijayakumar S., Ranganathan K., Kalyanasundaram N., Vanangamudi G., Thirunarayanan G., International Letters of Chemistry, Physics and Astronomy 4 (2014) 48-65. 